MIT Confidential

\title{
A Synthetic Transcription Platform for Programmable Gene Expression in Mammalian Cells
}

William C.W. Chen ${ }^{1,2,3,4, *}$, Leonid Gaidukov ${ }^{1,3, *}$, Ming-Ru Wu", ${ }^{1, \#}$, Jicong Cao ${ }^{1}$, Gigi C.G. Choi ${ }^{1, \# \#, ~ R a c h e l ~ P . ~}$ Utomo $^{1,5}$, Ying-Chou Chen ${ }^{1,2, \# \#, ~ L i l i a n a ~ W r o b l e w s k a ~}{ }^{6}$, Lin Zhang ${ }^{7}$, Ron Weiss ${ }^{1,3}$, Timothy K. Lu ${ }^{1,2,3, \&}$

${ }^{1}$ Synthetic Biology Center, Massachusetts Institute of Technology, Cambridge, MA, 02139, USA

${ }^{2}$ Research Laboratory of Electronics, Massachusetts Institute of Technology, Cambridge, MA, 02139, USA

${ }^{3}$ Department of Biological Engineering, Massachusetts Institute of Technology, Cambridge, MA, 02139, USA

${ }^{4}$ Cardiovascular Research Center, Massachusetts General Hospital, Boston, MA 02114, USA

${ }^{5}$ Department of Biochemistry, Wellesley College, Wellesley, MA 02481, USA

${ }^{6}$ Pfizer Inc., Cambridge, MA, 02139, USA

7 Pfizer Inc., Andover, MA, 01810, USA

\# Current address: Dana-Farber Cancer Institute and Harvard Medical School, Boston, MA 02215, USA

\#\# Current address: Laboratory of Combinatorial Genetics and Synthetic Biology, School of Biomedical Sciences, The University of Hong Kong, Hong Kong, China

\#\# Current address: Department of Life Sciences and Institute of Genome Sciences, National Yang-Ming University, Taipei, Taiwan

${ }^{*}$ Authors with equal contribution

\& To whom correspondence should be addressed. Tel: 617-715-4808; Email: timlu@mit.edu 
MIT Confidential

\section{Abstract (150 words)}

Precise, scalable, and sustainable control of genetic and cellular activities in mammalian cells is key to developing precision therapeutics and smart biomanufacturing. We created a highly tunable, modular, versatile CRISPR-based synthetic transcription system for the programmable control of gene expression and cellular phenotypes in mammalian cells. Genetic circuits consisting of well-characterized libraries of guide RNAs, binding motifs of synthetic operators, transcriptional activators, and additional genetic regulatory elements expressed mammalian genes in a highly predictable and tunable manner. We demonstrated the programmable control of reporter genes episomally and chromosomally, with up to 25-fold more EF1 $\alpha$ promoter activity, in multiple cell types. We used these circuits to program secretion of human monoclonal antibodies and to control T cell effector function marked by interferon- $\gamma$ production. Antibody titers and interferon- $\gamma$ concentrations were significantly correlated with synthetic promoter strengths, providing a platform for programming gene expression and cellular function for biological, biomanufacturing, and biomedical applications.

Key Words: Synthetic biology, Synthetic gene circuit, CRISPR/Cas9, RNA-guided gene regulation, Programmable gene expression, Synthetic transcription factor, Synthetic promoter, Antibody Production, Biomanufacturing, Precision Medicine 
MIT Confidential

\section{Introduction}

The regulation of gene expression in complex organisms has been a central focus for characterizing disease variation [1], producing monoclonal antibodies (mAbs) [2], and developing cell therapies. Synthetic biology offers powerful ways to harness artificial gene regulatory tools in mammalian systems [3-7]. For example, tumor-specific synthetic gene circuits applied to cancer immunotherapy yield anti-tumor responses in mice [8]. However, the use of strong constitutive promoters in gene expression platforms can increase the expression of target genes to the point of causing unwanted, dose-dependent side effects, raising safety concerns [9]. For instance, the use of the cytomegalovirus (CMV) promoter (CMVp) to express a tumor-killing gene markedly increases apoptosis in normal cells and induces acute systemic toxicity in vivo [10].

Extensive efforts have demonstrated the utility of tuning promoter activity with artificial transcription factors (TFs) derived from zinc fingers [11], TALEs [12], and CRISPR/Cas9 [13, 14], conjugated with DNA-binding, transcriptional activation domains (TADs). For example, several types of CRISPR-based TFs (crisprTFs) and compound activation mediators have been widely used in mammalian cells, such as dCas-VP16/-VP64/-VPR [15] and synergistic activation mediator (SAM) [16]. Our group also developed crisprTFs to regulate gene expression driven by natural and artificial eukaryotic promoters [17]. Here, we build upon those platforms by creating a crisprTF promoter system for the programmable regulation of gene expression in mammalian cells. Specifically, we created modular libraries of both crisprTFs and their synthetic operators by: 1) altering guide RNA (gRNA) sequences; 2) adjusting the number of gRNA binding sites (BS) in the synthetic operator; 3) incorporating additional control elements in the operator or crisprTF to augment expression; and 4) designing multiple orthogonal crisprTFs.

To demonstrate the utility of this synthetic transcription platform, we first validated the precise control of two reporter genes (mKate and BFP). We then used this system to produce recombinant human mAbs, including a functional checkpoint antibody, anti-human programmed cell death protein 1 (anti-hPD1) [18]. High-yield, stable production was achieved by using crisprTF promoters within a multi-landing pad (multi-LP) DNA integration platform for integrase-mediated, site-specific integration and stable expression of large gene circuits in multiple stable genomic loci in mammalian cells [19]. Multi-LP DNA integration enables predictable integration, stable gene expression, and consistent long-term protein production [19, 20]. The modular assembly of our artificial gene regulation system into chromosomally integrated gene circuits enables the precise and scalable engineering of mammalian cells. Anti-hPD1 expressed with this system can modulate certain anti-tumor phenotypes of human T cells. These results indicate that highly tunable, sustainable, and predictable protein expression over a wide dynamic range can be achieved with our synthetic transcription system.

\section{MATERIALS AND METHODS}

\section{Molecular Cloning and Genetic Circuit Construction}

All genetic circuits in this study were constructed using a modular Gateway-Gibson assembly method [21, 22]. Briefly, gRNAs and related sequences were commercially synthesized (IDT and GenScript) and then cloned into corresponding entry vectors using an InFusion approach (Takara, Inc) or a one-step Gibson 
MIT Confidential

reaction with in-house mastermix. Other genetic parts were cloned into corresponding entry vectors using the same approach. Multi-site LR reactions were performed between a promoter entry vector flanked by attL4 and attR1 recombination sites, a gene entry vector flanked by attL1 and attL2 recombination sites, and pZDonor_Seq $(n)$-GTW-Seq $(n+1) \_R 4$ R2 destination vectors containing a Gateway cassette (chloramphenicol resistance and $\mathrm{ccdB}$ genes flanked by attR4 and attR2 recombination sites) to generate positional expression vectors [i.e., independent transcription units (TUs) in different position vectors used for the subsequent Gibson assembly]. Gibson reactions were performed with the Gibson Assembly Ultra Kit (SGI-DNA) at $50^{\circ} \mathrm{C}$ for $60 \mathrm{~min}$, using equimolar concentrations (approximately 40-60 fmol per 10 ul reaction) of column-purified positional expression vectors (cleaved with I-Scel), a matching adaptor vector (cleaved with Xbal and Xhol), and a carrier vector with BxB1-attB or BxB1-GA-attB integration sites (cleaved with Fsel). Gibson-assembled constructs were diluted at 1:4 and used to transform E. coli 10G electrocompetent cells (60080-2, Lucigen). Cells were selected with appropriate selection antibiotics on solid and liquid culture; plasmids were column-purified with QIAprep Spin Miniprep Kit (27106, Qiagen). After verification by restriction mapping analysis and Sanger sequencing of payload TUs, correctly assembled constructs were expanded in $25 \mathrm{~mL}$ liquid culture with Stbl3 chemically competent cells (C737303, Invitrogen) and column-purified with QIAGEN Plasmid Plus Midi Kit (12945, Qiagen). A schematic diagram of the gene circuit construction methodology is shown in Figure 1A. Human elongation factor-1 alpha (EF1 $\alpha)$ promoter and mouse CMVp were used as constitutive promoter controls. Sp-dCas9-VPR was a gift from George Church (Addgene plasmid 63798) [23]. pRRL.CMVenh.gp91.Syn.Intron.eGFP was a gift from Didier Trono (Addgene plasmid 30470), which was used for cloning the 230-bp synthetic intron sequence [24].

\section{Landing Pad Vector Construction}

LP donor vectors for stable CRISPR-Cas9-mediated integration into the Chinese hamster ovary $(\mathrm{CHO})$ cell genome were constructed as previously reported [19]. Briefly, homology arm sequences for LP1-2, LP2, LP8, LP15, and LP20 sites (each arm approximately 0.5-1 kb long) were synthesized as a single gBlock (Integrated DNA Technologies) containing a Pmel restriction site between the left and right homology arms and unique Bsal cleavage sites in the 5' and 3' termini for Golden Gate cloning. Each gBlock was cloned into a pIDTsmart vector modified to contain compatible Bsal cloning sites. LP cassettes containing hEF1 $\alpha$-attP-BxB1-EYFP-P2A-Hygro (cassette 1), hEF1 $\alpha$-attP-BxB1-EBFP-P2A-Bla (cassette 2), or hEF1 $\alpha$-attP-BxB1-GA-EYFP-P2A-Hygro (cassette 3 ) were constructed using modular Gateway-Gibson cloning as previously described [21, 22]. LP cassettes were cloned into Pmel-linearized pIDTsmart backbones between the left and right homology arms using in-fusion cloning (Takara Bio USA). Using this approach, we generated the following LP donor vectors: LP1-2-cassette 1, LP2-cassette 1, LP2-cassette 2, LP8-cassette 2, LP15-cassette 2, LP20-cassette 2, and LP20-cassette 3 [19, 25].

\section{Cell Culture and Plasmid Transfection}

Adherent wild-type $\mathrm{CHO}-\mathrm{K} 1$ cells (CCL-61, ATCC) and engineered $\mathrm{CHO}$ cells were maintained in complete HAMS-F12 (cHAMS-F12) medium (ATCC) containing 10\% fetal bovine serum (FBS) (Sigma-Aldrich), 1\% HyClone non-essential amino acids (GE Healthcare Life Sciences), and 1\% penicillin/streptomycin (P/S) (Gibco by Life Sciences). Cells were grown in a humidified $37^{\circ} \mathrm{C}$ incubator with $5 \% \mathrm{CO}_{2}$ and passaged every 2-3 days. Transfections were carried out with the Neon electroporation system (Invitrogen) with $10 \mu$ Neon 
MIT Confidential

tips. Briefly, $1 \times 10^{5}$ cells were suspended in $\mathrm{R}$ buffer and mixed well with $250 \mathrm{ng}$ of individual experimental and transfection marker plasmids for 10-15 min at room temperature (RT). To ensure that all samples had the same total amount of plasmids, we supplemented the negative or positive control samples with a dummy plasmid composed of an identical expression vector backbone and a non-functional insert sequence. Cells were then electroporated with the setting of $1560 \mathrm{~V} / 5 \mathrm{~ms} / 10$ pulses. Transfected cells were immediately transferred to a 24-well plate containing $1 \mathrm{~mL}$ complete culture medium without any anti-microbial reagents. Fluorescence-activated cell sorting (FACS) analysis was performed at 48 hours post-transfection. Experiments were performed with independently transfected biological triplicates.

Human embryonic kidney (HEK)-293T cells (CRL-3216, ATCC) and mouse C2C12 myoblasts (CRL-1772, ATCC) were purchased from ATCC. Both cell types were thawed and expanded according to the manufacturer's instructions and subsequently maintained in Dulbecco's Modified Eagle medium (DMEM; Gibco by Life Sciences) containing 10\% FBS and 1\% P/S. Transfections were carried out with ViaFect transfection reagent (E4982, Promega). Briefly, cells were washed once with PBS, trypsinized, and re-suspended with complete culture medium at $1 \times 10^{5}$ cells per $100 \mu \mathrm{L}$. Experimental and transfection marker plasmids (250 ng each) were added in $100 \mu \mathrm{L}$ plain Opti-MEM medium and mixed well with ViaFect at a 1:3 ratio (total DNA by weight : ViaFect by volume), according to the manufacturer's recommendation. After incubating at RT for $10 \mathrm{~min}, 1 \times 10^{5}$ cells were added into the plasmid/ViaFect mixture and mixed well. Transfected cells were immediately transferred to a 24-well plate containing $0.8 \mathrm{~mL}$ complete culture medium without any antimicrobial reagents. FACS analysis was performed at 48 hours post-transfection.

\section{Engineering Landing Pad Cells}

Single- and multi-LP cell lines with engineered LP1-2, LP2, LP8, LP15, and LP20 loci were constructed in adherent $\mathrm{CHO}-\mathrm{K} 1$ cells by homologous recombination with CRISPR/Cas9 as described [19]. Briefly, targeted integrations were performed by co-transfecting $500 \mathrm{ng}$ of circular LP donor vector with $40 \mathrm{ng}$ of px330-U6-chimeric_BB-CBh-hSpCas9 vector, a gift from Feng Zhang (Addgene plasmid 42230) [26], and $150 \mathrm{ng}$ of U6-gRNA GeneArt DNA String (ThermoFisher). Roughly $10^{5}$ cells were transfected in triplicate with the Neon electroporation system with $10 \mu \mathrm{l}$ Neon tips and seeded in 24-well plate. Cells were then transferred to a six-well plate at 3 days post-transfection and subjected to antibiotic selection with either hygromycin $(600 \mu \mathrm{g} / \mathrm{ml})$ or blasticidin $(10 \mu \mathrm{g} / \mathrm{ml})$ for two weeks, followed by clonal cell sorting with FACS. Clonal cells were verified with diagnostic PCR using locus-specific and LP-specific primers (for on-target integration) and backbone-specific primers (for off-target integration). Single-cell clones exhibiting locus-specific and backbone-free integration, as well as stable and homogenous LP expression, were expanded, banked, and subsequently used for gene circuit integration.

\section{Chromosomal Integration of gene circuits}

For chromosomal integration of gene circuits in adherent, engineered $\mathrm{CHO}$ cells, cells were independently transfected in triplicate with $500 \mathrm{ng}$ of the BxB1 integrase-expressing plasmid (pEXPR-CAG-BxB1) and 500 ng DNA of the payload plasmid, using the Neon electroporation system with the same setting as described above. Transfected cells were immediately transferred to a 24-well plate containing $1 \mathrm{~mL}$ complete culture medium per well without any antimicrobial reagents. At 3 days post-transfection, cells were transferred to a 
MIT Confidential

6-well plate with $3 \mathrm{~mL}$ complete culture medium per well, followed by one of the following cell selection strategies (depending on the individual payload construct designs desribed in the Results section and figures): 1) for single integrants in single LP (sLP), cells were selected with puromycin only $(8 \mu \mathrm{g} / \mathrm{ml})$ for gene circuits with a single selection marker, or with both puromycin $(8 \mu \mathrm{g} / \mathrm{ml})$ and blasticidin $(10 \mu \mathrm{g} / \mathrm{mL})$ for gene circuits with both selection markers; 2) for single integrants in double LP (dLP), cells were selected with either puromycin $(8 \mu \mathrm{g} / \mathrm{mL})$ alone or with hygromycin $(250 \mu \mathrm{g} / \mathrm{mL})$ and $\mathrm{G} 418(250 \mu \mathrm{g} / \mathrm{mL})$ for the payload integrated in the LP2 locus, and with blasticidin $(10 \mu \mathrm{g} / \mathrm{mL})$ for the non-integrated, empty LP15 locus; 3) for double integrants in dLP, cells were selected with puromycin ( $8 \mu \mathrm{g} / \mathrm{mL})$, blasticidin $(10 \mu \mathrm{g} / \mathrm{mL})$, hygromycin $(250 \mu \mathrm{g} / \mathrm{mL})$, and G418 $(250 \mu \mathrm{g} / \mathrm{mL})$. Following 10 days of selection, correct integration was confirmed by FACS, indicated by the complete disappearance of native LP fluorescence (either EYFP, or EBFP, or both). Cells with chromosomally integrated gene circuits were maintained as pooled populations after selection. Cell viability and density were monitored with a Vi-CELL automated cell viability analyzer (Beckman-Coulter).

\section{Fluorescent Imaging}

All fluorescent images were taken with the EVOS FL Auto cell imaging system equipped with multiple LED light cubes, including TagBFP, GFP, YFP, RFP, and Texas Red (Thermo Fisher Scientific). Fluorescent images of the same color were taken with the same exposure settings. Scale bars were directly printed in the images.

\section{Flow Cytometry and Cell Sorting}

Cells were analyzed with a LSRFortessa flow cytometer, equipped with 405, 488, and $561 \mathrm{~nm}$ lasers (BD Biosciences). Thirty thousand events per sample were collected for analysis of median signal intensity of the entire transfected population, using a $488 \mathrm{~nm}$ laser and 530/30 nm bandpass filter for EYFP and a $405 \mathrm{~nm}$ laser, 450/50 filter for EBFP. Sphero rainbow calibration particles, 8 peaks (Spherotech) were used for instrument normalization and MEFL calculation. Median fluorescent intensity of the entire transfected population (i.e., all cells positive for both experimental and transfection marker signals) was measured. Data were analyzed with FACSDiva software (BD Biosciences), FlowJo, and FCS Express 6. Flow cytometry data were normalized to the EF1 $\alpha$ control and presented as relative median signal intensity (\%). Cell sorting was performed on a FACSAria cell sorter in the Swanson Biotechnology Center Flow Cytometry Facility at the Koch Institute (Cambridge, MA, USA). Untransfected CHO-K1 cells and unintegrated single- and multi-LP cells were used to set the gating. Different selection and sorting schemes were applied to target payload integration into specific LP sites, including: 1) single positive/single negative (EYFP+/EBFP- or EYFP-/EBFP+), 2) double-negative (EYFP-/EBFP-), or 3) double-negative/mKate-positive (EYFP-/EBFP-/mKate+) to select multi-LP cells with the payload(s) integrated into each designated LP. For pooled cell sorting, all sorted cells were initially seeded at 5,000-10,000 cells/cm in 24-well plates with $1 \mathrm{~mL}$ complete culture medium containing no antibiotics. For clonal cell sorting, single cells were initially sorted into flat-bottom 96-well plates with $100 \mu \mathrm{L}$ complete culture medium containing no antibiotics for clonal expansion and subsequently selected and expanded in 24-well and 6-well plates and T75 flasks.

\section{Human Antibody Production and Detection}


MIT Confidential

dLP-CHO cell lines were first integrated with a genetic circuit expressing only one copy of dCas-VPR. Cells were selected with hygromycin $(250 \mu \mathrm{g} / \mathrm{mL})$ and $\mathrm{G} 418(250 \mu \mathrm{g} / \mathrm{mL})$ for 10 days, followed by FACS sorting into a pool of single-positive cells using the LP-fluorescent reporters. Sorted cells were then integrated with a second, crisprTF-driven gene circuit expressing a human mAb, either JUG444 (Pfizer) or an anti-hPD1 (5C4) [8]. Double integrated cells were selected for 10 days with puromycin $(8 \mu \mathrm{g} / \mathrm{mL})$, blasticidin $(10 \mu \mathrm{g} / \mathrm{mL})$, hygromycin $(250 \mu \mathrm{g} / \mathrm{mL})$, and G418 $(250 \mu \mathrm{g} / \mathrm{mL})$, followed by FACS sorting into a pool of double-negative cells using the LP-fluorescent reporters. All experiments were performed with 3 independently transfected biological replicates. For mAb expression analysis, $1.5 \times 10^{5}$ cells were seeded in 24-well plate with $0.5 \mathrm{ml}$ cHAMS-F12 medium per well without antibiotics and maintained at $37^{\circ} \mathrm{C}$ for 4 days. Conditioned media were collected on day 4 for measurements. For long-term mAb expression analysis, master cultures were maintained and passaged accordingly for up to 5 weeks with measurements performed on the same day weekly. The amount of secreted JUG444 or anti-hPD1 was measured in duplicate with the Octet RED96 system using Protein A biosensors (ForteBio). Purified JUG444 or anti-hPD1 was used to generate a standard curve, from which the mAb titers were derived.

\section{CHO-Tumor-T Cell Co-Culture Model and Anti-tumor Activity Analysis}

dLP-CHO cells with an integrated gene circuit expressing anti-hPD1 (5C4) were seeded into a 12-well plate in triplicate at a density of $1 \times 10^{5}$ cells/well with $1 \mathrm{~mL} \mathrm{CHO}$ cell culture medium. Cells were incubated at $37^{\circ} \mathrm{C}$ for $48 \mathrm{hrs}$. Pre-activated human T cells and human ovarian cancer cells (OVCAR8) expressing a surface T cell engager [8] were mixed at an effector-to-target ( $\mathrm{E}: \mathrm{T})$ ratio of $20: 1\left(1 \times 10^{6}: 0.5 \times 10^{5}\right.$ cells) in $1 \mathrm{~mL}$ T cell culture medium (RPMI-1640 with 10\% FBS, 1\% P/S, $10 \mathrm{mM}$ HEPES, $0.1 \mathrm{mM}$ non-essential amino acids, $1 \mathrm{mM}$ sodium pyruvate, , and $50 \mu \mathrm{M}$ 2-Mercaptoethanol) and then added to each well containing attached dLP-CHO cells. Co-culture of three cell types (CHO, OVCAR8, and T cells) were incubated at $37^{\circ} \mathrm{C}$. Cell-free supernatants were collected and processed at $24 \mathrm{hrs}$ and stored at $-20^{\circ} \mathrm{C}$. As an indicator of the $\mathrm{T}$ cell response to tumor cells in the presence of actively secreted anti-hPD1 from dLP-CHO cells, interferon (IFN)- $\gamma$ produced by $\mathrm{T}$ cells was quantified. IFN- $\gamma$ concentrations in cell-free supernatants were determined by Human IFN- $\gamma$ DuoSet enzyme-linked immunosorbent assay (ELISA; DY285; R\&D systems, Minneapolis, MN, USA).

\section{Statistical Analysis}

All quantitative data are presented as mean \pm standard deviation (SD). Statistical differences between groups were analyzed by Student's $t$-test (for two groups), one-way ANOVA (for multiple groups), or two-way ANOVA (for long-term stability measurements) with 95\% confidence interval. Statistical significance was set at $p \leqq 0.05$. Dunnett test was performed to correct for multiple comparisons in ANOVA post-hoc analysis. All statistical analyses, including correlation analysis and linear regression, were performed and graphed with GraphPad Prism 7.0 (GraphPad Software, La Jolla, CA, USA) statistics software.

\section{Results}

\section{Construction of a programmable, modular synthetic transcription system with crisprTFs}

To enable high tunability and versatility of the synthetic transcription system for a wide spectrum of 
MIT Confidential

applications, we adopted a 3-tiered modular library design [21] with a variety of interchangeable gene regulatory parts (Figure $1 \mathrm{~A}$ ). We built a library of guide RNAs (gRNAs) that were orthogonal to the $\mathrm{CHO}$ genome and first selected 8 top scored ones for evaluation (Figure 1B). For each gRNA, we designed a corresponding operator containing $8 x$ complementary gRNA binding sites (BS) to drive the expression of a reporter gene, mKate, and evaluated their performance episomally (Figure 1C and Supplemental Figure 1). Flow cytometry results showed a wide range of mKate expression levels among different gRNAs (Figure 1D). As active natural mammalian promoter sequences typically have a high GC content (57\%) [27], we suspected that the GC content of the gRNA seed sequence (8-12 bases at the 3' end of a gRNA and its BS sequence) plays a role in regulating gene expression. gRNAs with around $50-60 \%$ GC in seed sequences appeared to express mKate at higher levels (Figure 1B and 1D). Therefore, we selected a relatively weak gRNA (gRNA9) with a high GC content $(\geq 70 \%)$ and introduced mutations in 2 consecutive bases within the seed sequence to alter its GC content. The resulting gRNA10 and its matching operator, with $\geq 50 \%$ GC and still orthogonal to the $\mathrm{CHO}$ genome, yielded much higher mKate expression than its ancestor, gRNA9 (Figure 1D, $p=0.0004$ ). The CMV control and gRNA1, 2, 6, 7, and 10 had higher expression (Figure 1D, all $p \leq 0.0005$ ), while only gRNA4 had lower expression, than the EF1 $\alpha$ control (Figure $1 \mathrm{D}, p<0.05$ ). The expression obtained with gRNA1, 2 , and 10 was not significantly different from that obtained with the CMV control (all $p>0.05$ ). Overall, the top four gRNAs, with more than 2 -fold higher expression than EF1 $\alpha$, had $50-60 \%$ GC in the first 8-10 bases of the seed sequences (Supplemental Figure 2). We selected three representative gRNAs that yielded weak (gRNA4), medium (gRNA7), and strong expression (gRNA10) to investigate further. A comparison of three crisprTFs (dCas-VP16, dCas-VP64, dCas-VPR) showed that dCas-VPR yielded a markedly higher expression level than dCas-VP16 or dCas-VP64 (Supplemental Figure 3 , both $p<0.0001$ ), consistent with a previous report [15]. Thus, to enable the largest dynamic expression range possible, we selected dCas-VPR for subsequent investigation.

\section{Transcriptional programming in multiple mammalian cell types}

To control gene expression at distinct levels, we built a library of synthetic operators containing 2x-16x gRNA BS for gRNA4, 7, and 10 (Figure 1C). Transient expression of mKate was assessed in CHO cells at 48 hrs post-transfection (Supplemental Figure 4). Flow cytometry data showed dramatically different patterns of mKate expression among the three gRNA series (Figure 2A). The gRNA4 series ranged from 15\% (2x BS) to $270 \%$ (16x BS) of EF1 $\alpha$, with $2 x-8 x$ BS driving notably lower, and 16x BS driving significantly higher, expression than the EF1 $\alpha$ control. The gRNA7 series ranged from $26 \%$ (2x BS) to $760 \%$ (16x BS) of EF1 $\alpha$, with $2 x-4 x$ BS driving notably lower, and $8 x-16 x$ BS driving significantly higher, expression than EF1 $\alpha$. The gRNA10 series ranged from $30 \%$ (2x BS) to $1107 \%$ (16x BS) of EF1 $\alpha$, with $6 x-16 x$ BS having significantly higher expression than EF1 $\alpha$ (Figure 2A). Significant correlations between expression levels and the number of BS were found with all three gRNA series (Supplemental Figure $5 A$, all $p<0.05$ ). Overall, with differences in gRNA sequences and the number of complementary gRNA BS in the operators, we achieved a wide dynamic range of approximately 74 -fold change in the intensity of the reporter signals with these gRNA series in $\mathrm{CHO}$ cells.

To translate these results into other mammalian cell types (mouse and human), we selected the gRNA10 series because it had the lowest leakage and the highest expression among the three comprehensively 
MIT Confidential

tested gRNAs. Interestingly, we observed dramatic differences in mKate expression with the CMV control in mouse $\mathrm{C} 2 \mathrm{C} 12$ myoblasts (Figure 2B) and human HEK cells (Figure 2C). The gRNA10 series behaved in a similar order in these cells as it did in $\mathrm{CHO}$ cells. In C2C12 cells, mKate expression ranged from 11\% (2x BS) to $316 \%$ (16x BS) of EF1 $\alpha$, with 2x-6x BS being notably lower and 8x-16x BS being significantly higher than EF1 $\alpha$ (Figure 2B). In HEK cells, mKate expression ranged from 60\% (2x BS) to $1026 \%$ (16x BS) of EF1 $\alpha$, with $6 x-16 x$ BS being significantly stronger than EF1 $\alpha$ (Figure $2 \mathrm{C}$ ). Similarly, expression levels and the number of BS markedly correlated in $\mathrm{C} 2 \mathrm{C} 12$ and HEK cells with the gRNA10 series (Supplemental Figure 5B-C, all $p \leq 0.0005)$. Collectively, with the gRNA10 series alone, we achieved approximately 29 -fold and 17-fold changes in mKate expression in mouse and human cells, respectively.

\section{Incorporation of additional genetic control elements to enhance gene expression}

To maximize expression of the target gene, we incorporated the following additional genetic control elements in the crisprTF promoters: (i) synergistic activation mediator (SAM); (ii) dual gRNA transcriptional units (TUs); (iii) an additional 2x nuclear localizing sequence (NLS) at the 5' end of dCas-VPR; and (iv) a synthetic intron ( $\mathrm{SI}$ ) to enhance post-transcriptional processing (Figures 3A-D). Transient mKate expression in CHO-K1 cells was assessed at 48 hrs post-transfection. The combination of SAM, dCas-VPR, and the gRNA10 8x BS operator resulted in a $48 \%$ increase in gene expression $(p=0.0003)$; however, gene expression decreased by $12.3 \%$ when SAM was combined with dCas-VPR and the 16x BS operator ( $p=0.0023$ ), suggesting a limitation of gene activation by conjoining various TADs (Figure 3A). With the addition of an extra gRNA10 TU to boost the amount of gRNA, we observed modest increases, of $18.7 \%$ $(p=0.0278)$ and $17.5 \%(p=0.009)$, with gRNA10 $8 x$ and 16x BS operators, respectively (Figure 3B). Thus, a single gRNA TU may be sufficient for many applications. With an additional $2 x$ NLS incorporated at the 5 ' end of dCas-VPR, mKate expression increased by $64.0 \%(p=0.0055)$ and $33.0 \%(p=0.0034)$ with gRNA10 $8 \mathrm{x}$ and $16 \mathrm{x}$ BS operators, respectively, suggesting that the nuclear localization of the original dCas-VPR may be slightly insufficient to gain maximum activation of gene expression (Figure 3C).

On the other hand, the addition of SI at the 5' untranslated region (UTR) of the target gene led to an approximately $200-300 \%$ elevation in mKate signals with nearly all operators in all three gRNA series when compared with their original counterparts, even with the strongest operator (i.e., 16x BS of gRNA10; Supplemental Figure 6). The gRNA4-SI series yielded 70\% (4x BS+SI) to 528\% (16x BS+SI) expression levels of the EF1 $\alpha$ promoter, with $8 x-16 x$ BS+SI being notably higher than the EF1 $\alpha$ control; the gRNA7-SI series ranged from $65 \%(4 \mathrm{x} \mathrm{BS}+\mathrm{SI})$ to $532 \%(16 \mathrm{xS}+\mathrm{SI})$ of $\mathrm{EF} 1 \alpha$, with $8 \mathrm{x}-16 \mathrm{x} \mathrm{BS}+\mathrm{SI}$ being significantly higher than EF1 $\alpha$; the gRNA10-SI series ranged from 205\% (4x BS+SI) to 2463\% (16x BS+SI) of EF1 $\alpha$, with $8 \mathrm{x}-16 \mathrm{x}$ BS+SI showing significantly higher expression than EF1 $\alpha$ (Figure 3D). We did not observe any substantial increases of fluorescent signals in the absence of gRNA in any of the SI constructs (Figure 3D). These results indicated that $\mathrm{SI}$ can be fully compatible with synthetic crisprTF promoter-based gene regulation and is an efficient control element to increase gene expression. Notable correlations were found between expression levels and the number of BS with the gRNA4-SI and gRNA10-SI series (Supplemental Figure 7 , both $p<0.05$ ). Taken together, by adding $S I$ to the original gRNA operators, we expanded the achievable dynamic expression range to an approximately 167-fold change in $\mathrm{CHO}$ cells, offering analogue precision control of gene expression with a near-continuous spectrum (Supplemental Figure 8). 
MIT Confidential

\section{Precision control of gene expression via genomic integration}

We designed our crisprTF promoter platform to be fully compatible with our multi-LP DNA integration platform [19] for the stable expression of large gene circuits in engineered mammalian cells. We built gene circuits that contained insulated TUs encoding different gRNAs, gRNA operator-target gene pairs, and a crisprTF, together with an integration-enabled circuit selection marker, puromycin (Figure 4A). Chromosomal integration of a single DNA copy of the gene circuit was mediated by BxB1 integrase into a single LP (sLP) locus in engineered adherent $\mathrm{CHO}$ cells (Figure 4A). After selection with puromycin for integration in engineered SLP-CHO cells, indicated by the disappearance of the EYFP signal, we analyzed target gene expression by flow cytometry. Both the gRNA4 and gRNA10 series exhibited tunable control of expression profiles similar to that seen with their episomal counterparts (Figure 4B). Gene expression of the gRNA4 series ranged from $7 \%(2 x \mathrm{BS})$ to $56 \%$ (16x BS) of the integrated EF1 $\alpha$ control, with 2x-6x BS exhibiting markedly lower expression than EF1 $\alpha$; the gRNA10 series ranged from 13\% (2x BS) to $207 \%$ (16x BS) of EF1 $\alpha$, with $2 x-4 x$ BS also exhibiting significantly lower expression than EF1 $\alpha$. Adding $\mathrm{SI}$ at the 5' UTR resulted in 200-250\% increases in target gene expression with representative integration circuits (Figure 4B, right side of the panel). With a single genomically integrated copy, only gRNA10 16x BS without SI (207\%) and $8 \mathrm{x}$ and $16 \mathrm{x}$ BS with SI (223\% and 521\% respectively) exhibited significantly stronger expression than the integrated EF1 $\alpha$ control (Figure 4B). Similar to what was observed with their episomal counterparts when both the gRNA4 and gRNA10 series were chromosomally integrated into SLP, the correlations between the number of BS in the synthetic operator and gene expression levels were highly significant (Supplemental Figure 9A; both $p<0.005$ ).

Nonetheless, by observing the expression profiles of puromycin-selected pools of crisprTF circuit integrants, we found that after 4 weeks of culturing, all four circuits from both the gRNA4 and gRNA10 series had notably decreased expression levels, suggesting the occurrence of silencing events (Supplemental Figure $9 \mathrm{~B}$, all $p<0.05$ at 4 weeks post selection). To prevent silencing of the transcriptional activator and improve long-term expression profiles, we incorporated an additional 3' flanking selection marker, blasticidin, into dCas-VPR, linked by a $2 \mathrm{~A}$ self-cleavage peptide (Figure 4C). Flow cytometry results demonstrated gene expression levels that were similar to slightly higher with most integration circuits immediately after dual selection, compared with their unmodified counterparts (Figure 4D). Unlike the CMV control (Figure 4E, $p<0.0001$ at 2 and 4 weeks), observations over four weeks revealed improved stability in expression levels, with no marked change in any of the four circuits examined (Figure $4 \mathrm{E}$, all $p>0.05$ at 2 and 4 weeks). Collectively, these data suggested that potential crisprTF circuit silencing post-integration in a SLP may be prevented by using a flanking selection marker co-expressed with the dCas-VPR gene.

\section{Modulation of human monoclonal antibody production}

The controllable production of mAbs is desirable for many biomedical applications. To determine whether our transcriptional platform could be used for the precise control of antibody synthesis, we built integration gene circuits expressing a human mAb, JUG444, with the light chain (LC) and heavy chain (HC) genes separately expressed by the same gRNA10 operator as independent TUs (Figure 5A). We had previously observed that dCas9-VPR could occasionally be silenced when expressed at high levels under the control of 
MIT Confidential

CMVp (data not shown). To avoid the silencing of dCas9-VPR during long-term culture, we used adherent double LP (dLP)-CHO cells, engineered with two distinct wild-type LPs: dLP1-1 and dLP1-2, to accommodate an additional copy of the dCas9-VPR gene (Figure 5A) [20, 28]. We first performed a targeted integration into dLP1-1 alone in dLP-CHO cells with a DNA payload encoding dCas9-VPR (Figure 5A). Cells were selected with three antibiotics and then subjected to single-cell FACS to isolate EYFP-/EBFP+ clones with the stably integrated dLP1-1 site and the free dLP1-2 site.

The functionality of clonally expanded cells was examined by integrating individual gRNA10 control circuits that co-expressed mKate and BFP reporter genes, as well as another copy of the dCas9-VPR gene, as independent TUs into the dLP1-2 site (Figure 5A). Dually integrated cells were selected with four antibiotics and then FACS-sorted into pools to expand them and evaluate reporter expression (Figure 5A). We found that mKate and BFP driven by the same gRNA10 operators were simultaneously expressed at similar levels in two distinct configurations (8x BS without $\mathrm{SI}$ and 16x BS with $\mathrm{SI}$ ), suggesting uniform transcriptional activation of both reporter TUs by two separate copies of the dCas-VPR gene (Figure 5B). Next, we replaced the control circuit in the dLP1-2 site with individual gRNA10 mAb circuits that differentially express JUG444 (Figure 5A). Similarly, cells with mAb circuits were selected and FACS-sorted into pools.

To examine the precision regulation and long-term stability of mAb production, we maintained cultures of sorted pools over five weeks and measured mAb concentration weekly. We recorded differential JUG444 production in Week 1, driven by four distinct configurations: 8x or 16x BS, with or without SI (JUGAb1-4, Figure $5 \mathrm{C}$ ). Stable mAb production from a single mAb gene copy was sustained throughout the experimental duration with all four configurations (Figure $5 \mathrm{C}$ ). Strikingly, mAb production levels significantly correlated with gRNA10 operator strengths documented in Figure 4D (Figure 5D, $p<0.05$ at all time points). The strongest operator (16x BS with SI) yielded the highest mAb level, which was comparable to the level previously observed with the strong constitutive CMVp expressing two mAb gene copies [19]. Overall, these data indicated that tunable and lasting control can be applied to express human antibodies and potentially other therapeutic proteins.

\section{Programmable control of the human T cell immune response against tumor cells}

To explore applications of our platform for cellular therapy, we built gRNA10 integration circuits with different configurations to program the secretion of anti-hPD1, an important immune checkpoint inhibitor widely used as a therapy in immuno-oncology [18]. The light chain and heavy chain of anti-hPD1 were separately expressed by the same gRNA10 operators as independent TUs (Figure 6A). Similar to the JUG444 production, a copy of dCas9-VPR was pre-integrated into the dLP1-1 site of the dLP-CHO cells to avoid its complete silencing. Each of the four circuits, designed to differentially express anti-hPD1 (8x or 16x BS, with or without SI), was then integrated into the dLP1-2 site of the dLP-CHO cells, which had been selected and clonally sorted for dLP1-1 occupancy (PD1Ab1-4, Figure 6B). Dually integrated cells were selected and then sorted into pools with FACS for expansion. Quantification of anti-hPD1 secretion by sorted cells showed that the PD1Ab4 group (16x BS with SI) had a significantly higher titer of anti-hPD1 than all the other groups (Figure $6 \mathrm{~B}$, all $p<0.001$ ), and the PD1Ab3 group (16x BS without $\mathrm{SI}$ ) had a notably higher titer than the PD1Ab1 group (8x BS without SI) (Figure 6B, p<0.05). A strong correlation was found between anti-hPD1 
MIT Confidential

titers and crisprTF promoter strengths (Figure $6 \mathrm{C}, p<0.005$ ).

To demonstrate the functionality of actively secreted anti-hPD1, we developed a CHO-tumor-T cell co-culture system (Figure 6D). We hypothesized that differentially programmed anti-hPD1 secretion would enhance $T$ cell effector function by correspondingly blocking the interactions between tumor cells and T cells, mediated by the engagement of PD1 with programmed death ligands. Sorted, dually integrated dLP-CHO cells with individual configurations expressing anti-hPD1 were first seeded. Pre-activated human T cells and ovarian cancer cells expressing a surface-displayed T-cell engager were then added to the attached dLP-CHO cells [8]. IFN- $\gamma$ production by T cells was measured at 24 hours post-co-culture as a marker of T cell activation (Figure 6D). T cells in the PD1Ab3 and PD1Ab4 groups produced significantly more IFN- $\gamma$ than the control group ( $p<0.05$ and $p<0.01$ respectively), corresponding to the higher titers of anti-hPD1 secretion (Figure 6E).

\section{Discussion}

We have built and characterized a crisprTF promoter system for the programmable regulation of gene expression in mammalian cells. This system functions consistently across multiple mammalian cell types and is, therefore, sufficiently versatile to be used in a variety of cellular models for biomedicine and biomanufacturing. To demonstrate the generalizability of the system's gene regulatory activity, we used episomal vectors in transiently transfected cells and site-specific, stably integrated chromosomal constructs in LP-engineered cells. We modulated three key parameters: 1) the gRNA sequence; 2) the number of gRNA BS repeats in the operator; and 3) the CRISPR-based transcriptional activator (different crisprTFs, different selection markers in the crisprTF, or an additional copy of the crisprTF in LP-engineered cells). We also incorporated genetic elements to synergistically enhance gene expression, including SAM, extra gRNA TU, extra NLS, and $\mathrm{SI}$ in the operator. Systematic characterization of these constructs resulted in gene expression constructs with $>1,000$-fold range of expression levels. The strongest synthetic promoters, composed of 16x gRNA BS repeats, were significantly stronger than CMVp, one of the strongest constitutive promoters used for mammalian applications [29]. The introduction of a 2-bp mutation that moderately altered the GC ratio in the seed sequence of gRNA10 and its BS markedly changed its gene expression profiles, suggesting the important role of the gRNA seed sequence in controlling crisprTF-based transcriptional activities. In future work, we anticipate that additional large-scale experimental screening, combined with computational modeling or machine learning, could be used to program gene expression even more precisely.

Previous research has identified various mechanisms that control gene expression at the transcriptional level in eukaryotes [30]. Core promoters and neighboring genetic elements can impact gene expression because of their instrumental roles in consolidating complex cascades of the signaling events involved in transcription [30]. A previous report showed positive transcriptional elements situated between -350 and -40 bp relative to the transcription start sites (TSS) in many of the tested human promoters, whereas negative regulatory elements were more likely to be located -350 to -1000 bp upstream of the TSS [27]. Therefore, we designed our synthetic operators to be consistent with natural promoters (up to roughly -400 bp upstream of the TSS). 
MIT Confidential

The results of our episomal tests with all three gRNA series established strong linear correlations between gene expression levels and the number of BS (up to 16x) in the operators (Supplemental Figures 5 and 7). We found similar significant correlations with two gRNA series when they were genomically integrated in LP-engineered cells (Supplemental Figure 9A). Overall, our data are consistent with previous reports suggesting that longer transcriptional bursts and thus higher expression levels might be achieved with synthetic transcriptional activators by arraying multiple BS upstream of a given promoter [17, 31].

In addition to tuning transcriptional strengths by altering gRNA sequences and operator strengths, we discovered several compatible genetic control elements that synergistically augment gene expression. When combined with dCas-VPR, SAM moderately increased expression at medium, but not high, levels episomally. We further examined the compatibility of SAM with dCas-VPR when genomically integrated by assembling large gRNA circuits that contained independent TUs encoding both systems (Supplemental Figure 10A). Surprisingly, the VPR-SAM hybrid system at medium expression level generated no further increase in gene expression; in fact, at high expression level, VPR-SAM actually decreased expression (Supplemental Figure 10B). This seemingly paradoxical result suggested possible competition for transcriptional resources among components within the hybrid system, for example, overlapping TADs encoded in SAM and dCas-VPR genes, including VP64 and p65 [32, 33]. On the other hand, introns have been proposed to control post-transcriptional RNA processing or transport [34] and have been shown to enhance gene expression in mammalian cells when applied as a positive regulatory element [35]. Here, the incorporation of SI immediately downstream of the operator in the 5' UTR of the target gene [36] resulted in an increase in gene expression of at least $200 \%$ with nearly all tested constructs, whether episomal or chromosomal. These results suggested a high degree of compatibility between the transcriptional control mediated by the crisprTF promoter and the post-transcriptional regulation mediated by SI. To enable exogenously modulated gene expression, we incorporated two types of small molecule-inducible switches, leveraging TetR and Lacl, to control gRNA expression in the episomal context (Supplemental Figure 11A). The results indicate that an additional layer of gene tunability can be achieved in the crisprTF promoter system with the addition of small molecule-inducible switches (Supplemental Figure 11B-C).

Recombinant therapeutic proteins are increasingly important to the pharmaceutical industry and clinical medicine [37]. $\mathrm{CHO}$ cells, widely used for biomanufacturing therapeutic proteins and approved by regulatory agencies [37], are easily cultured in suspension and produce high titers of therapeutic proteins with human-like glycosylation profiles [38]. CMVp and Simian virus 40 early promoter are among the constitutive promoters most frequently used in $\mathrm{CHO}$ cells for recombinant protein production [39]. Yet, protein-producing cell lines often exhibit a wide range of gene expression profiles and unstable or silenced gene expression, possibly due to the ill-defined mechanisms that drive viral promoters in eukaryotic cells [40]. Commonly used natural promoters have often evolved synchronously with functionalities that depend on specific genetic contexts, so promoter activity may not transfer across various genes and species [30]. Thus, the growing number of biotherapeutic proteins in development has created an increasing demand for an efficient, constitutive gene expression system for biomanufacturing, ideally with built-in programmable control of protein production for improved consistency and predictable yields. In this study, we demonstrated the long-term stability of precisely tuned human mAb production with the crisprTF promoter system. Yields of 
MIT Confidential

antibody production obtained with the strongest crisprTF circuit expressing a single mAb LC/HC cassette were similar to those obtained with two or six mAb cassettes driven by CMV or EF1 $\alpha$ promoters, respectively, in LP-engineered CHO cells [19]. The high production efficiency of our strong crisprTF circuits, along with their built-in modularity and scalability, suggests that they can be adapted, with further improvement, to achieve industrially relevant $\mathrm{mAb}$ titers. These results also suggest that our crisprTF promoter platform can be applied, more generally, to $\mathrm{CHO}$ strains optimized for industrial bioreactor conditions for increased target protein production in a controlled manner.

In conclusion, we have achieved tunable, scalable, and sustainable gene expression by building a well-characterized system of crisprTF-based regulatory elements. This platform should enable programmable, multiplexed gene modulation for broad applications, such as mammalian synthetic biology, biomanufacturing, and precision medicine.

\section{Acknowledgements}

The authors gratefully thank Nevin M. Summers and Dr. Louane Hann for their administrative support, Kristjan E. Kaseniit for his contribution in gRNA design, Dr. Kevin J. Lebo for his expert input in the system design, Dr. Samuel D. Perli for the genetic materials, and Dr. Wen Allen Tseng for the genetic materials and his contribution in the construct design. We greatly thank Selamawit Mamo, Kalpana Jagtap, Na Li, Cong Liu, and Jonathan L. Lyles for their technical support with $\mathrm{CHO}$ cell cultures and assays, Mailing Ding and Christina Harrison for their technical support with molecular cloning and plasmid preparation, and Karen Pepper for editing the manuscript. We thank Dr. G. Felsenfeld (National Institute of Diabetes and Digestive and Kidney Diseases, USA) for the HS4 insulator sequences.

\section{Funding}

This work was supported by the Pfizer-MIT collaboration. W.C. was supported in part by the NIH Ruth L. Kirschstein NRSA postdoctoral fellowship (5T32HL007208).

\section{Data availability}

The authors declare that all relevant data supporting the findings of this study are available within the paper and its supplementary Information. Biological materials generated in this study are available on Addgene or from the corresponding author upon request.

\section{Competing interests}

T.K.L. is a co-founder of Senti Biosciences, Synlogic, Engine Biosciences, Tango Therapeutics, Corvium, BiomX, and Eligo Biosciences. T.K.L. also holds financial interests in nest.bio, Ampliphi, IndieBio, MedicusTek, Quark Biosciences, and Personal Genomics. All other authors declare no competing interests.

\section{References}

[1] Consortium GT, Aguet F, Brown AA, Castel SE, Davis JR, He Y, et al. Genetic effects on gene expression across human tissues. Nature. 2017;550:204.

[2] Shukla AA, Thömmes J. Recent advances in large-scale production of monoclonal antibodies and related 
MIT Confidential

proteins. Trends in Biotechnology. 2010;28:253-61.

[3] Courbet A, Renard E, Molina F. Bringing next-generation diagnostics to the clinic through synthetic biology. EMBO Mol Med. 2016;8:987-91.

[4] Higashikuni Y, Chen WCW, Lu TK. Advancing therapeutic applications of synthetic gene circuits. Current Opinion in Biotechnology. 2017;47:133-41.

[5] Lillacci G, Benenson Y, Khammash M. Synthetic control systems for high performance gene expression in mammalian cells. Nucleic Acids Research. 2018;46:9855-63.

[6] Matsuura S, Ono H, Kawasaki S, Kuang Y, Fujita Y, Saito H. Synthetic RNA-based logic computation in mammalian cells. Nature Communications. 2018;9:4847-.

[7] Mansouri M, Strittmatter T, Fussenegger M. Light-Controlled Mammalian Cells and Their Therapeutic Applications in Synthetic Biology. Adv Sci (Weinh). 2018;6:1800952-.

[8] Nissim L, Wu M-R, Pery E, Binder-Nissim A, Suzuki HI, Stupp D, et al. Synthetic RNA-Based Immunomodulatory Gene Circuits for Cancer Immunotherapy. Cell. 2017;171:1138-50.e15.

[9] Chen C, Yue D, Lei L, Wang H, Lu J, Zhou Y, et al. Promoter-Operating Targeted Expression of Gene Therapy in Cancer: Current Stage and Prospect. Molecular Therapy - Nucleic Acids. 2018;11:508-14. [10] Xie X, Li L, Xiao X, Guo J, Kong Y, Wu M, et al. Targeted Expression of BikDD Eliminates Breast Cancer with Virtually No Toxicity in Noninvasive Imaging Models. Molecular Cancer Therapeutics. 2012;11:1915-24.

[11] Klug A. The Discovery of Zinc Fingers and Their Applications in Gene Regulation and Genome Manipulation. Annual Review of Biochemistry. 2010;79:213-31.

[12] Moore R, Chandrahas A, Bleris L. Transcription activator-like effectors: a toolkit for synthetic biology. ACS Synthetic Biology. 2014;3:708-16.

[13] Doudna JA, Charpentier E. The new frontier of genome engineering with CRISPR-Cas9. Science. 2014;346:1258096.

[14] Kim T, Lu TK. CRISPR/Cas-based devices for mammalian synthetic biology. Current Opinion in Chemical Biology. 2019;52:23-30.

[15] Chavez A, Tuttle M, Pruitt BW, Ewen-Campen B, Chari R, Ter-Ovanesyan D, et al. Comparison of Cas9 activators in multiple species. Nat Meth. 2016;13:563-7.

[16] Konermann S, Brigham MD, Trevino AE, Joung J, Abudayyeh OO, Barcena C, et al. Genome-scale transcriptional activation by an engineered CRISPR-Cas9 complex. Nature. 2015;517:583-8.

[17] Farzadfard F, Perli SD, Lu TK. Tunable and Multifunctional Eukaryotic Transcription Factors Based on CRISPR/Cas. ACS Synthetic Biology. 2013;2:604-13.

[18] Havel JJ, Chowell D, Chan TA. The evolving landscape of biomarkers for checkpoint inhibitor immunotherapy. Nature Reviews Cancer. 2019;19:133-50.

[19] Gaidukov L, Wroblewska L, Teague B, Nelson T, Zhang X, Liu Y, et al. A multi-landing pad DNA integration platform for mammalian cell engineering. Nucleic Acids Research. 2018;46:4072-86.

[20] Jusiak B, Jagtap K, Gaidukov L, Duportet X, Bandara K, Chu J, et al. Comparison of Integrases Identifies Bxb1-GA Mutant as the Most Efficient Site-Specific Integrase System in Mammalian Cells. ACS Synthetic Biology. 2019;8:16-24.

[21] Guye P, Li Y, Wroblewska L, Duportet X, Weiss R. Rapid, modular and reliable construction of complex mammalian gene circuits. Nucleic Acids Research. 2013;41:e156. 
MIT Confidential

[22] Duportet X, Wroblewska L, Guye P, Li Y, Eyquem J, Rieders J, et al. A platform for rapid prototyping of synthetic gene networks in mammalian cells. Nucleic Acids Research. 2014;42:13440-51.

[23] Chavez A, Scheiman J, Vora S, Pruitt BW, Tuttle M, P R lyer E, et al. Highly efficient Cas9-mediated transcriptional programming. Nat Meth. 2015;12:326-8.

[24] Barde I, Laurenti E, Verp S, Wiznerowicz M, Offner S, Viornery A, et al. Lineage- and stage-restricted lentiviral vectors for the gene therapy of chronic granulomatous disease. Gene Therapy. 2011;18:1087. [25] Chang MM, Gaidukov L, Jung G, Tseng WA, Scarcelli JJ, Cornell R, et al. Small-molecule control of antibody N-glycosylation in engineered mammalian cells. Nature Chemical Biology. 2019.

[26] Cong L, Ran FA, Cox D, Lin S, Barretto R, Habib N, et al. Multiplex Genome Engineering Using CRISPR/Cas Systems. Science. 2013;339:819-23.

[27] Cooper SJ, Trinklein ND, Anton ED, Nguyen L, Myers RM. Comprehensive analysis of transcriptional promoter structure and function in 1\% of the human genome. Genome research. 2006;16:1-10.

[28] Inniss MC, Bandara K, Jusiak B, Lu TK, Weiss R, Wroblewska L, et al. A novel Bxb1 integrase RMCE system for high fidelity site-specific integration of mAb expression cassette in CHO Cells. Biotechnology and Bioengineering. 2017;114:1837-46.

[29] Qin JY, Zhang L, Clift KL, Hulur I, Xiang AP, Ren B-Z, et al. Systematic Comparison of Constitutive Promoters and the Doxycycline-Inducible Promoter. PLoS ONE. 2010;5:e10611.

[30] Leavitt JM, Alper HS. Advances and current limitations in transcript-level control of gene expression. Current Opinion in Biotechnology. 2015;34:98-104.

[31] Raj A, Peskin CS, Tranchina D, Vargas DY, Tyagi S. Stochastic mRNA Synthesis in Mammalian Cells. PLOS Biology. 2006;4:e309.

[32] Qian Y, Huang H-H, Jiménez JI, Del Vecchio D. Resource Competition Shapes the Response of Genetic Circuits. ACS Synthetic Biology. 2017;6:1263-72.

[33] Wilkinson AC, Nakauchi H, Göttgens B. Mammalian Transcription Factor Networks: Recent Advances in Interrogating Biological Complexity. Cell Systems. 2017;5:319-31.

[34] Rose AB. Introns as Gene Regulators: A Brick on the Accelerator. Frontiers in Genetics. 2019;9.

[35] Mariati, Ho SCL, Yap MGS, Yang Y. Evaluating post-transcriptional regulatory elements for enhancing transient gene expression levels in CHO K1 and HEK293 cells. Protein Expression and Purification. 2010;69:9-15.

[36] Choi T, Huang M, Gorman C, Jaenisch R. A generic intron increases gene expression in transgenic mice. Molecular and Cellular Biology. 1991;11:3070-4.

[37] Walsh G. Biopharmaceutical benchmarks 2018. Nature Biotechnology. 2018;36:1136.

[38] Yang Z, Wang S, Halim A, Schulz MA, Frodin M, Rahman SH, et al. Engineered CHO cells for production of diverse, homogeneous glycoproteins. Nature Biotechnology. 2015;33:842.

[39] Brown AJ, James DC. Precision control of recombinant gene transcription for $\mathrm{CHO}$ cell synthetic biology. Biotechnology Advances. 2016;34:492-503.

[40] Romanova N, Noll T. Engineered and Natural Promoters and Chromatin-Modifying Elements for Recombinant Protein Expression in CHO Cells. Biotechnology Journal. 2017;13:1700232. 
MIT Confidential

Figures and Figure Legends

Figure 1.

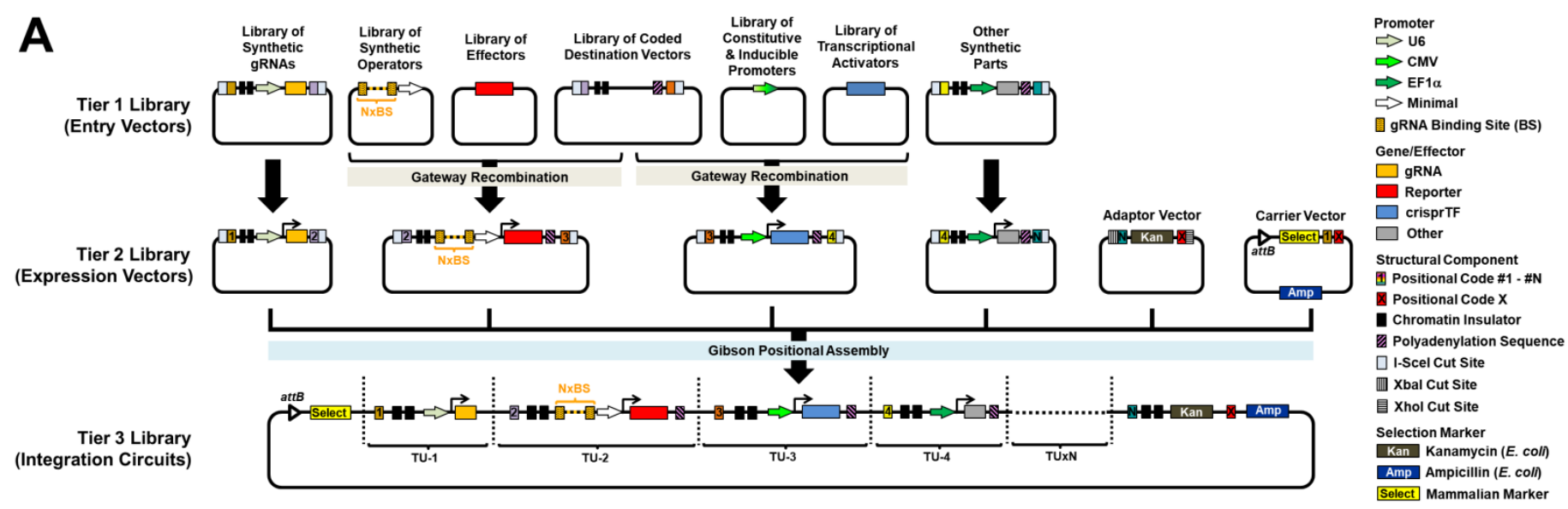

B

\begin{tabular}{|c|c|c|c|c|c|}
\hline Label & Sequence & $\begin{array}{c}\text { Total GC } \\
(\%)\end{array}$ & $\begin{array}{c}\text { Seed } 8 \text { bp } \\
\text { GC }(\%)\end{array}$ & $\begin{array}{c}\text { Seed } 10 \text { bp } \\
\text { GC }(\%)\end{array}$ & $\begin{array}{c}\text { Seed } 12 \text { bp } \\
\text { GC }(\%)\end{array}$ \\
\hline gRNA1 & ACCTCCGTTCAACTATCGC & 52.6 & 50.0 & 50.0 & 41.7 \\
gRNA2 & TAATCGCCTTCCGGACGAT & 52.6 & 62.5 & 60.0 & 58.3 \\
gRNA3 & ATCGTCTGCGATAGAACGA & 47.4 & 37.5 & 40.0 & 50.0 \\
gRNA4 & CGCGAGGGTAGTTGACAAC & 57.9 & 37.5 & 40.0 & 41.7 \\
gRNA5 & CGAACCTCTCGTTGCACGA & 57.9 & 50.0 & 60.0 & 58.3 \\
gRNA6 & TGGTTCGTAACGCCATAAC & 47.4 & 50.0 & 50.0 & 41.7 \\
gRNA7 & AGTACGGGTCGTACACGAA & 52.6 & 37.5 & 50.0 & 50.0 \\
gRNA8 & CGCGGGCAAGATCTTATAC & 52.6 & 25.0 & 30.0 & 25.0 \\
gRNA9 & TACGAGGGCGATCCGGCGA & 68.4 & 75.0 & 70.0 & 75.0 \\
gRNA10 & TACGAGGGCGATCCAACGA & 57.9 & 50.0 & 50.0 & 58.3 \\
\hline
\end{tabular}

C
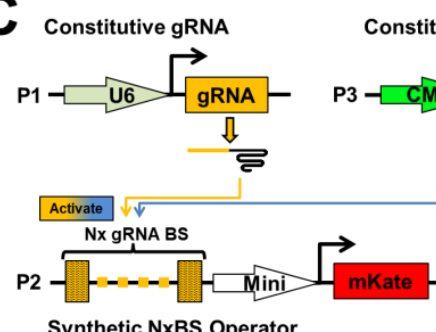

Constitutive crisprTF
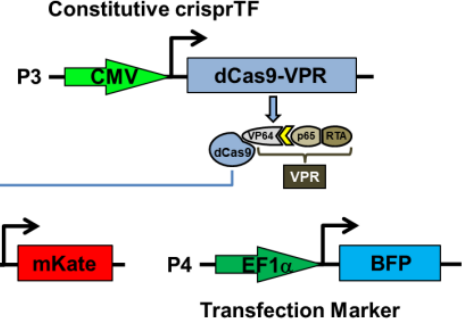

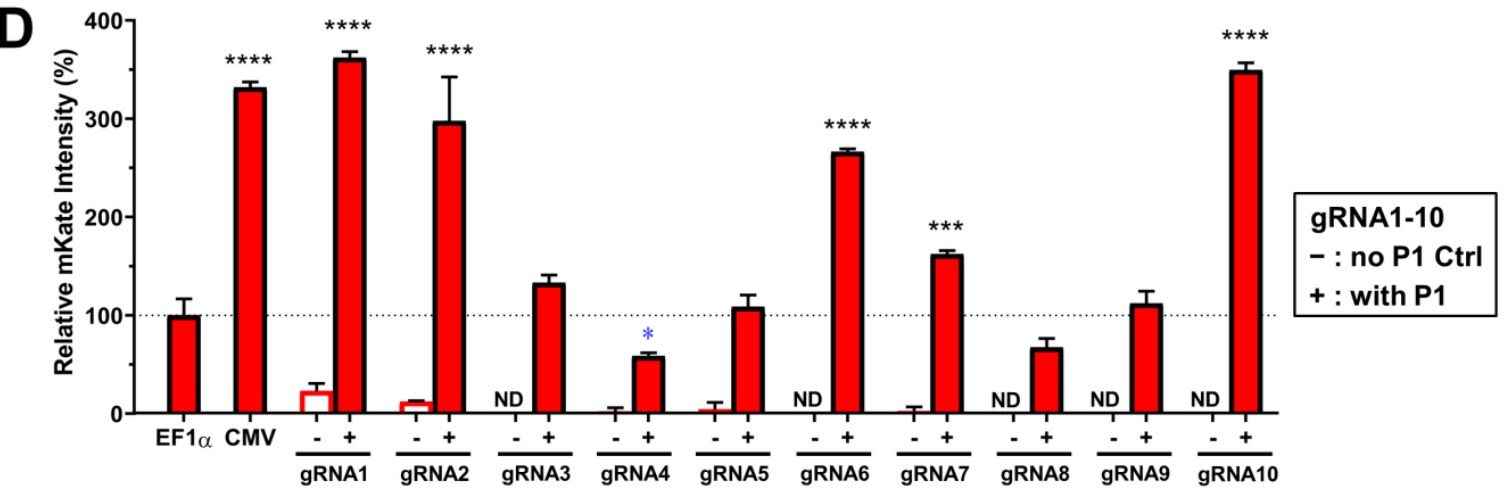

Figure 1. Design and development of the crisprTF promoter system.

(A) Schematic illustration of the programmable and modular design of the crisprTF promoter system. To increase its programmability, this platform was modularly divided into 3 tiers of libraries constructed with the Gateway-Gibson cloning approach. The Tier 1 library was composed of entry vector modules separately encoding gRNAs, synthetic operators with gRNA binding sites (BS) upstream of a minimal promoter, effector genes, crisprTFs and their associated promoters, and other transcriptional control elements. Tier 1 library units were assembled into positional expression vectors with pre-defined orders by Gateway cloning, forming the Tier 2 library. Positional assembly by Gibson cloning was performed to connect independent transcriptional units (TUs), derived from positional expression vectors in the Tier 2 library by I-Scel restriction digestion, into complete gene circuits. The Tier 3 library comprised integration circuits enabling precision control of the target gene(s) when integrated into a landing pad (i.e., a designated chromosomal safe harbor) with BxB1 integrase-mediated, site-specific chromosomal integration. (B) Ten gRNAs (gRNA1-10) 
MIT Confidential

orthogonal to the $\mathrm{CHO}$ genome were screened for expression. gRNA10 was modified from gRNA9 with GG-to-AA mutations to reduce the GC content of the seed and the entire sequences. (C) To evaluate episomal gene expression levels, $\mathrm{CHO}-\mathrm{K} 1$ cells were transiently transfected with four plasmids: plasmid \#1 (P1) constitutively expressing gRNA; plasmid \#2 (P2) encoding the synthetic operator with some number ( $x$ ) of gRNA BS to drive mKate expression; plasmid \#3 (P3) constitutively expressing a crisprTF; and plasmid \#4 (P4) constitutively expressing the transfection marker (BFP). mKate signals were assessed at 48 hrs post-transfection. (D) For the gRNA screening, each gRNA was paired with a matching synthetic operator containing 8x gRNA BS to control mKate expression. EF1 $\alpha$ and CMV promoters driving mKate expression served as positive controls. Experimental groups (+) were transfected with four plasmids (P1-P4). Control groups (-) to detect baseline operator leakage were transfected without P1 (P2-P4). Data were normalized to the EF1 $\alpha$ control and are presented as relative median mKate intensity (\%). gRNA1 and gRNA2 operators exhibited notable leakage without gRNA, suggesting non-specific transcriptional activities that were not associated with targeted crisprTF binding.

Figure 2.
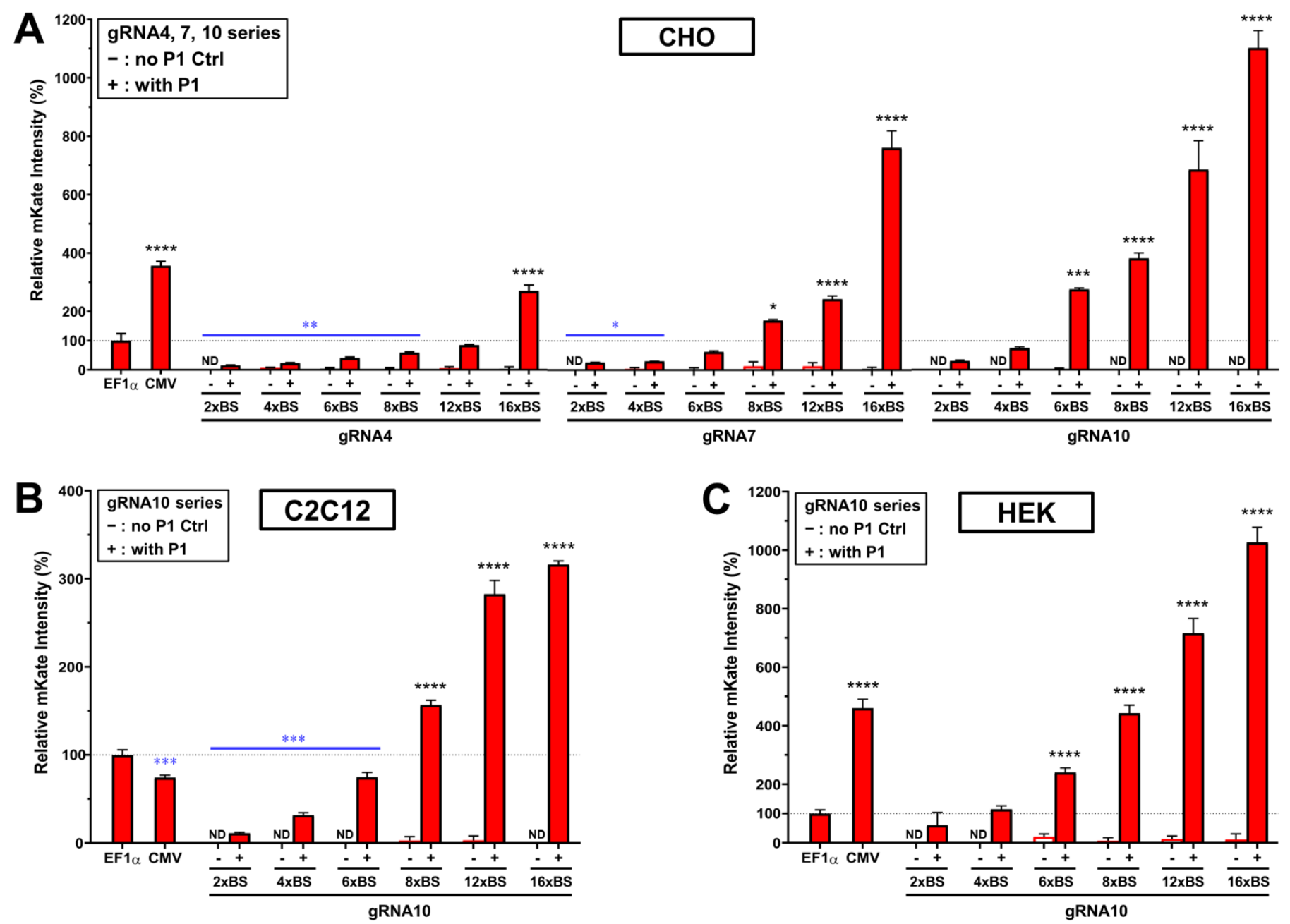

Figure 2. Gene expression programmed by the number of gRNA BS in synthetic operators.

A library of gRNA4, 7, and 10 synthetic operators containing 2x-16x gRNA BS was built to assess the number of $g R N A$ BS that would be effective as a programmable parameter in controlling gene expression levels. Experimental groups (+) were transiently transfected with four plasmids (P1-P4 in Figure 1C) 
MIT Confidential

whereas control groups (-) were transfected without P1 (only P2-P4 in Figure 1C) to detect operator leakage. $\mathrm{EF} 1 \alpha$ and CMV promoters served as positive controls. Median mKate signals relative to the EF1 $\alpha$ control were analyzed at $48 \mathrm{hrs}$ post-transfection with flow cytometry. (A) In CHO-K1 cells, synthetic operators of the gRNA4, 7, and 10 series with 2x-16x BS were examined. Overall, gRNA4 operators with 2x-8x BS and gRNA7 operators with $2 x-4 x$ BS had notably lower expression than the EF1 $\alpha$ control, whereas the gRNA4 operator with 16x BS, gRNA7 operators with 8x-16x BS, and gRNA10 operators with $6 x-16 x$ BS had significantly higher expression than the EF1 $\alpha$ control. (B) In mouse C2C12 myoblasts, gRNA10 operators with 2x-6x BS had notably lower expression than the EF1 $\alpha$ control, whereas operators with $8 x-16 x$ BS had significantly higher expression than the EF1 $\alpha$ control. (C) In human HEK-293T cells, the gRNA10 operators with $6 x-16 x$ BS had significantly higher expression than the EF1 $\alpha$ control. Red solid bars represent experimental groups (+); paired red hollow bars represent corresponding control groups without gRNA (-). ND: not detected; for increased expression (marked in black): ${ }^{*} p \leq 0.05$; ${ }^{* * *} p \leq 0.001 ;{ }^{* * *} p \leq 0.0001$; for

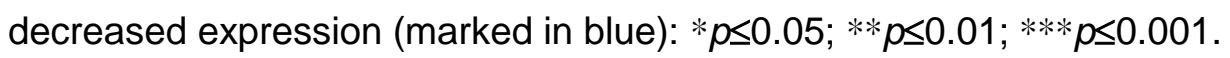

Figure 3.
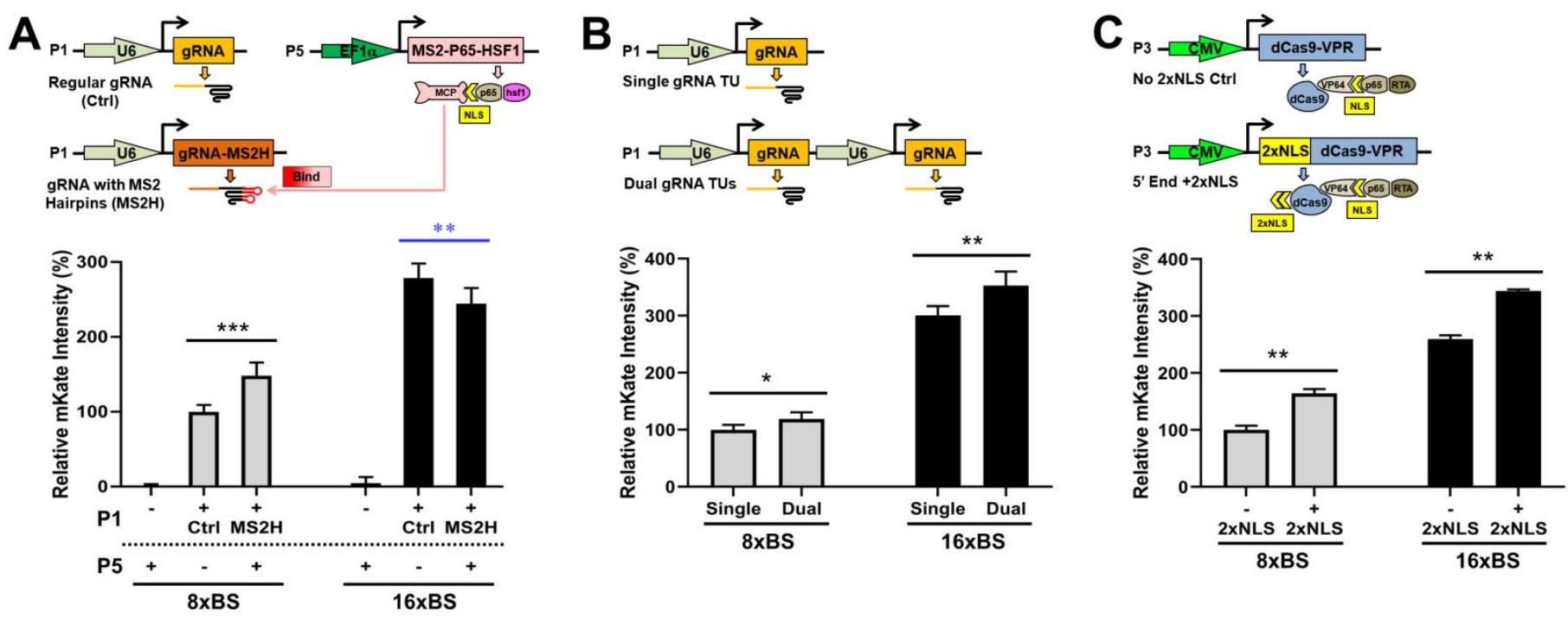

D

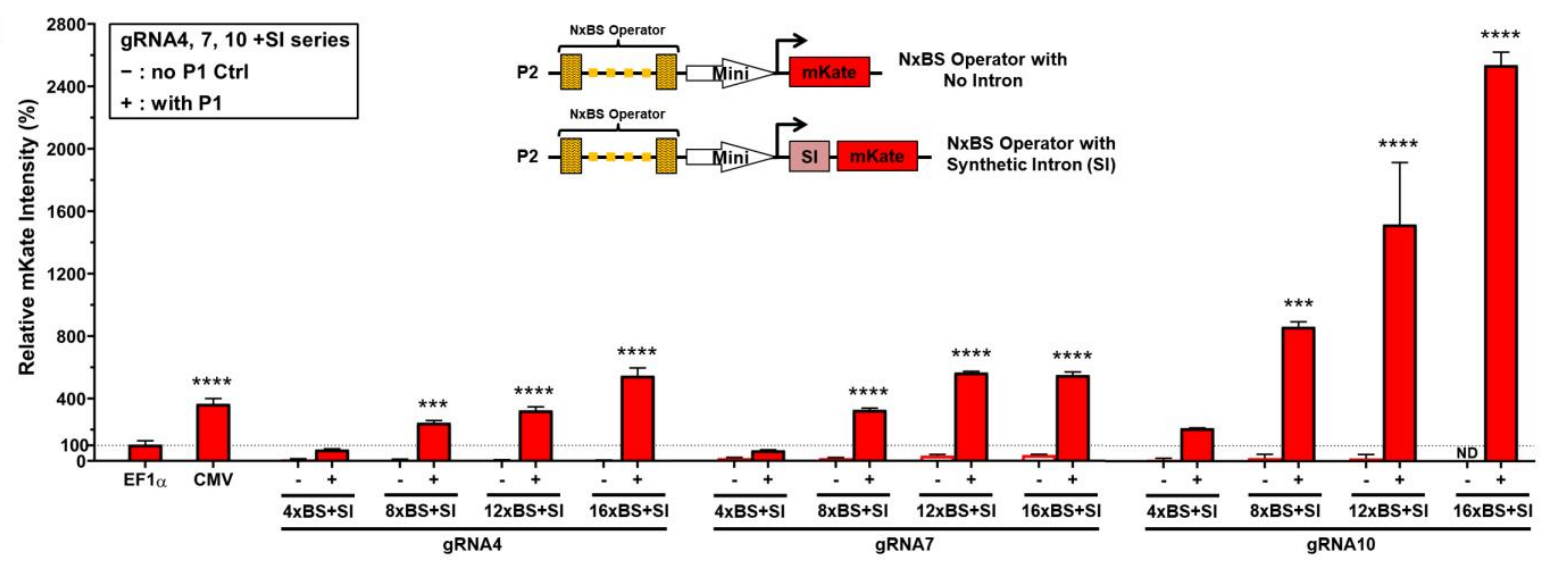

Figure 3. Genetic control elements to enhance gene expression.

To maximize the expression level of the target gene, additional genetic control elements were incorporated into the crisprTF promoters. Experimental groups (+) were transiently transfected with four or five plasmids 
MIT Confidential

(P1-P4 or P1-P5), while negative controls (-) were transfected without P1. Flow cytometry was performed at $48 \mathrm{hrs}$ post-transfection to analyze median mKate signal intensities relative to the EF1 $\alpha$ control. Schematic illustrations depicting individual experiments only exhibit plasmid constructs that were different from P1-P4 in Figure 1C. (A) Synergistic activation mediator (SAM). By combining dCas-VPR with SAM, another strong synthetic transcriptional activator, we investigated the feasibility of engineering a VPR-SAM hybrid system with a synergistic effect in gene expression. (B) Dual gRNA transcriptional units (TUs). By adding an extra gRNA10 TU to increase gRNA expression, we investigated whether the amount of gRNA was a limiting factor for target gene expression. (C) Additional 2x nuclear localizing sequence (NLS) in the crisprTF. We investigated whether adding extra 2x NLS at the 5' end of dCas-VPR would enrich nuclear localization of the crisprTF to increase target gene expression. (D) Synthetic intron (SI). By adding SI at the 5' UTR of the target gene, we investigated whether the crisprTF promoter-mediated transcriptional programming was compatible with SI-mediated post-transcriptional regulation. SI was incorporated into four synthetic operators of the gRNA4, 7 , and 10 series (4x, 8x,12x, and $16 x$ BS), respectively. The results showed that operators with $8 x-16 x \mathrm{BS}+\mathrm{SI}$ in all three gRNA-SI series drove markedly higher mKate expression than the EF1 $\alpha$ control. Control groups lacking gRNA had none or minimal leakage. Red solid bars represent experimental groups (+); paired red hollow bars represent corresponding control groups without gRNA (-). ND: not detected; for increased expression (marked in black): ${ }^{*} p \leq 0.05$; ${ }^{* *} p \leq 0.01$; ${ }^{* * *} p \leq 0.001 ;{ }^{* * *} p \leq 0.0001$; for decreased expression (marked in blue): ${ }^{* *} p \leq 0.01$ ) 
MIT Confidential

Figure 4.

A Adherent $\mathrm{CHO}$ Cells with Single Landing Pad (sLP)
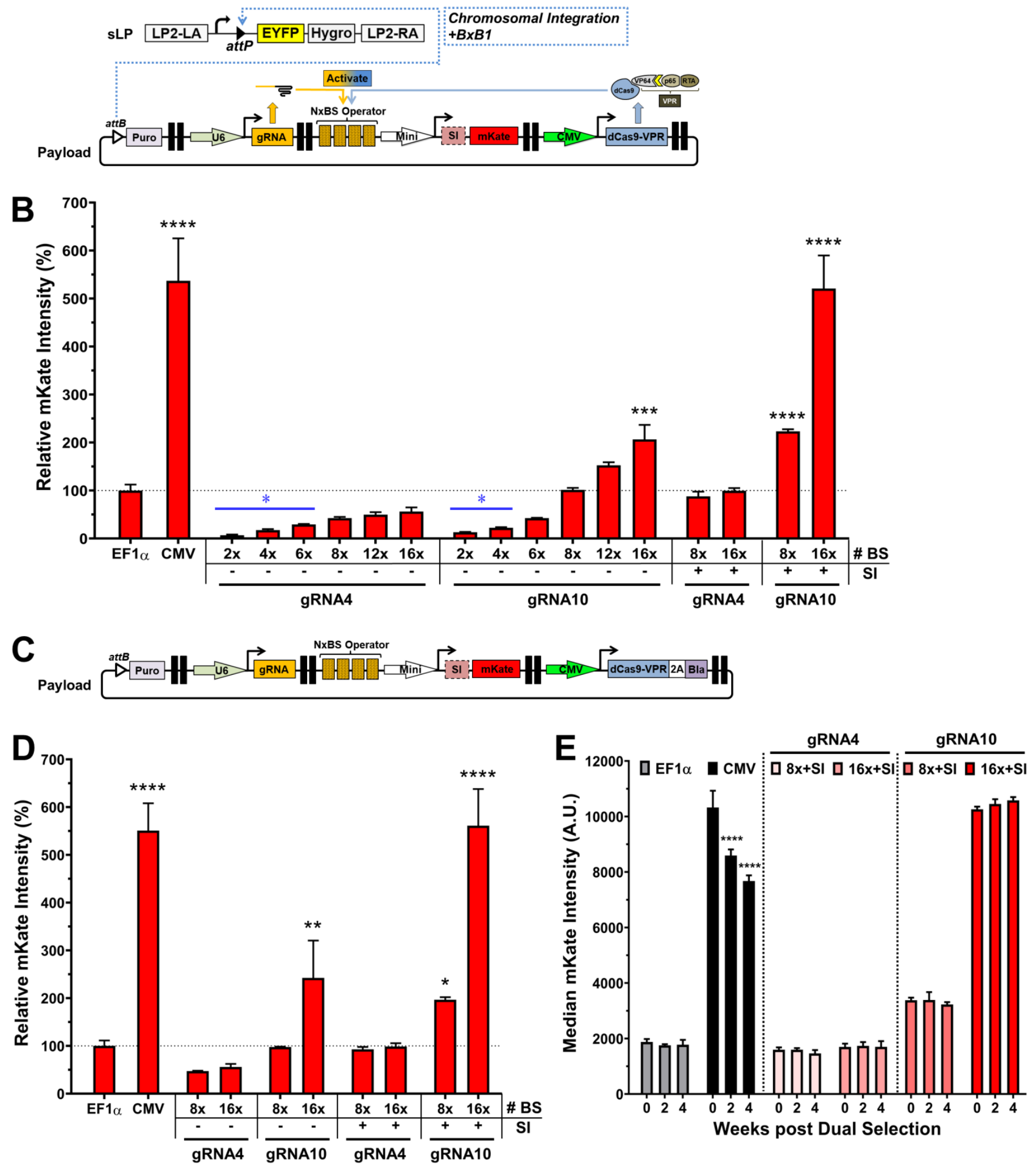

Figure 4. Genomic integration and long-term precision gene expression.

(A) A schematic illustration of an integration gene circuit and BxB1 recombinase-mediated, site-specific integration in an engineered, adherent $\mathrm{CHO}$ cell line with a single landing pad (sLP). Positive integration control circuits had a central TU with an EF1 $\alpha$ or CMV promoter driving mKate expression and two flanking dummy TUs with no gene expression in the same architecture. (B) After puromycin selection, flow cytometry was performed to assess median mKate signal intensities relative to the chromosomally integrated EF1 $\alpha$ 
MIT Confidential

control circuit. Overall, gRNA4 circuits with 2x-6x BS operators and gRNA10 circuits with 2x-4x BS operators exhibited significantly lower expression than the EF1 $\alpha$ control, whereas gRNA10 circuits with 16x BS, 8x $\mathrm{BS}+\mathrm{SI}$, and 16x BS+SI operators displayed considerably stronger expression than the EF1 $\alpha$ control. (C) A schematic illustration of an integration circuit with the addition of a 3' flanking selection marker (blasticidin) into dCas-VPR, linked by a 2A self-cleavage peptide, to prevent silencing in long-term culture. A number of integration circuits from the gRNA4 and gRNA10 series were selected for the modification. (D) Immediately after dual selection with puromycin and blasticidin, flow cytometry was performed to determine median mKate signal intensities. The data showed similar or slightly higher gene expression levels with most tested circuits when compared with their counterparts without the 3' flanking blasticidin. (E) We examined mKate expression levels with two of the strongest circuits from each gRNA series over the course of 4 weeks following dual selection. All four circuits examined displayed no noteworthy change in mKate signals at 2 or 4 weeks (all $p>0.05$ ), similar to the EF1 $\alpha$ control. In contrast, the CMV control exhibited notable decreases at both time points (both $p<0.0001$ ). 
MIT Confidential

Figure 5.

A Adherent CHO Cells with Double Landing Pad (dLP)
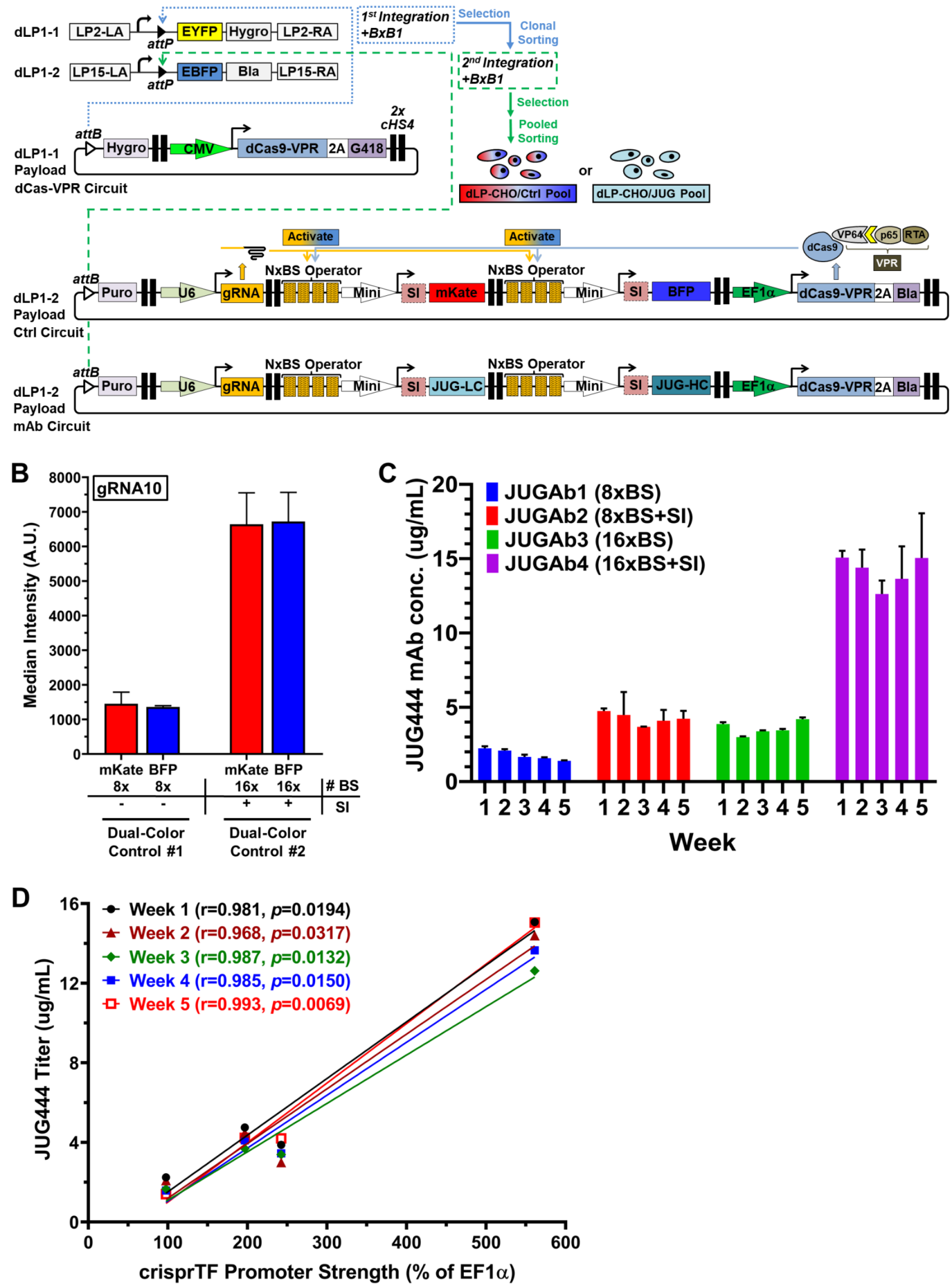
MIT Confidential

\section{Figure 5. Precision control of human monoclonal antibody (mAb) production.}

(A) Schematic illustrations of the sequential, site-specific genomic integration of two payload gene circuits into the $\mathrm{CHO}$ cells engineered with a double landing pad (dLP) for human mAb production. First, a synthetic gene circuit encoding one copy of dCas9-VPR and 2 flanking mammalian selection markers, hygromycin ( $5^{\prime}$ end) and G418 (3' end), was integrated into dLP1-1 site (LP2-attP-BxB1-EYFP-Hygro) with BxB1 integrase. Cells were subjected to a 10-day selection with hygromycin and G418 for the occupancy of the dLP1-1, as well as blasticidin for the free dLP1-2 site (LP15-attP-BxB1-EBFP-Bla). Selected cells were clonally sorted based on EYFP-/EBFP+ signals. Isolated clones were recovered with no antibiotics for one passage and then expanded under the same selective conditions. A single clone with the most consistent outputs of dCas9-VPR and EBFP was chosen for the second BxB1-mediated integration targeting the free dLP1-2 site. The second gene circuit integrated into the cells contained independent TUs encoding either mKate and BFP reporter genes (control circuit) or the light chain and heavy chain of a mock human mAb JUG444 (mAb circuit) as well as gRNA10, dCas9-VPR, and two additional flanking selection markers: puromycin (5' end) and blasticidin ( $3^{\prime}$ end). Integration of both circuits, in dLP1-1 and dLP1-2 sites respectively, was demonstrated by selecting cells with four antibiotics for two weeks and then subjecting them to pooled cell sorting based on EYFP-/EBFP- signals. Sorted cells with the stably integrated dLP1-1 and dLP1-2 sites were recovered for one passage and then expanded under the same selective conditions. (B) Using the same gRNA10 operators to express mKate and BFP individually, both genes had similar expression levels in two distinct configurations (8x BS without SI and 16x BS with SI). (C) Using the same gRNA10 operators to express the light chain and heavy chain of JUG444 individually, we examined mAb production with four configurations: JUGAb1 (8x BS), JUGAb2 (8x BS with SI), JUGAb3 (16x BS), and JUGAb4 (16x BS with SI). Octet mAb titer quantitation over five weeks showed stable, differential JUG444 production by all four integration circuits. (D) Pearson correlation analysis was performed to determine the relationship between JUG444 mAb titers and crisprTF promoter strengths reported in Figure 4D over the course of five weeks. The Pearson correlation coefficients $(r)$ at Week 1 through Week 5 were: $r=0.98\left(R^{2}=0.96, p=0.0194\right), r=0.97$ $\left(R^{2}=0.94, p=0.0317\right), r=0.99 \quad\left(R^{2}=0.97, p=0.0132\right), r=0.99 \quad\left(R^{2}=0.97, p=0.015\right)$, and $r=0.99 \quad\left(R^{2}=0.99\right.$, $p=0.0069)$, respectively. 
MIT Confidential

Figure 6.

A Adherent $\mathrm{CHO}$ Cells with Double Landing Pad (dLP)

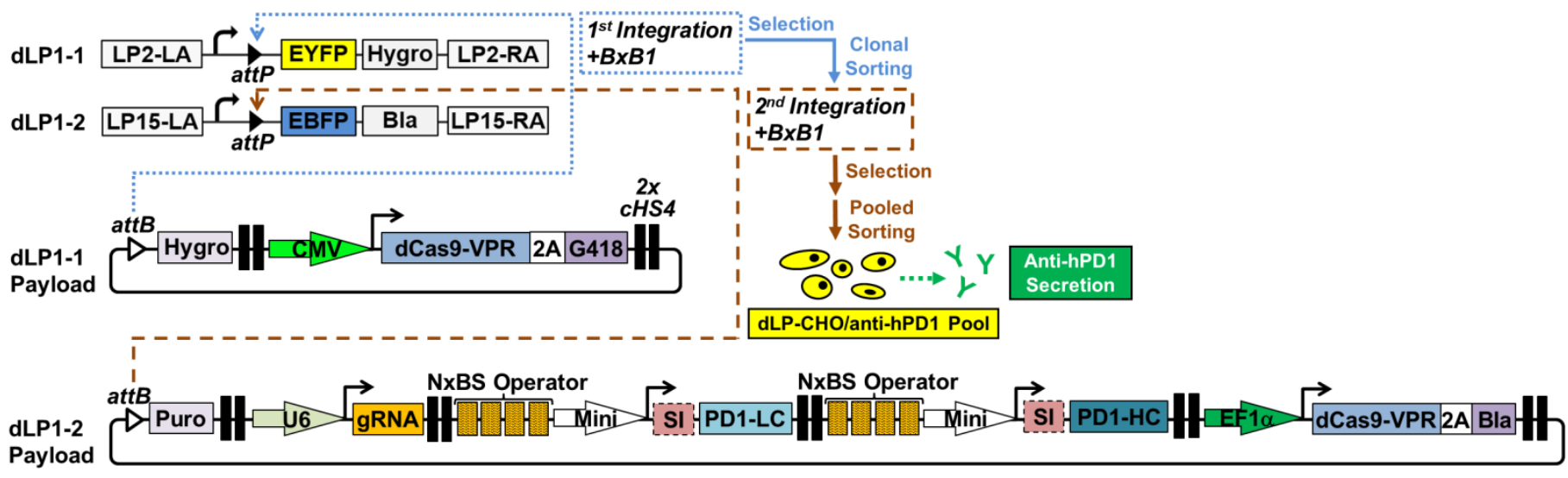

B

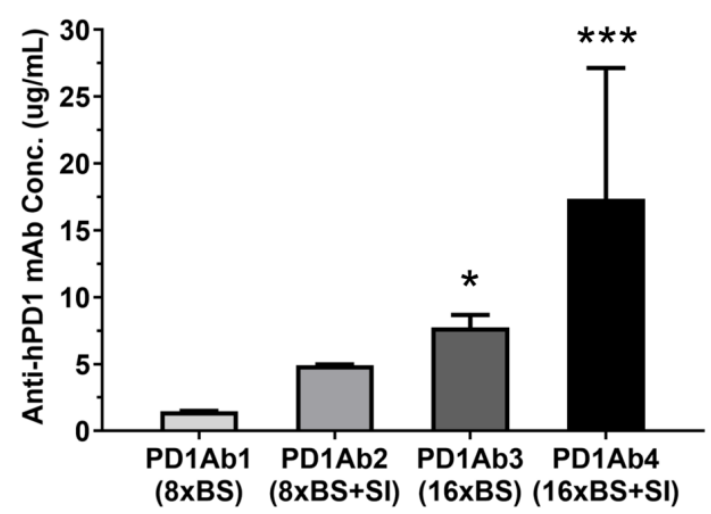

D

dLP-CHO/anti-hPD1 + Tumor cells + T cells Co-culture Model
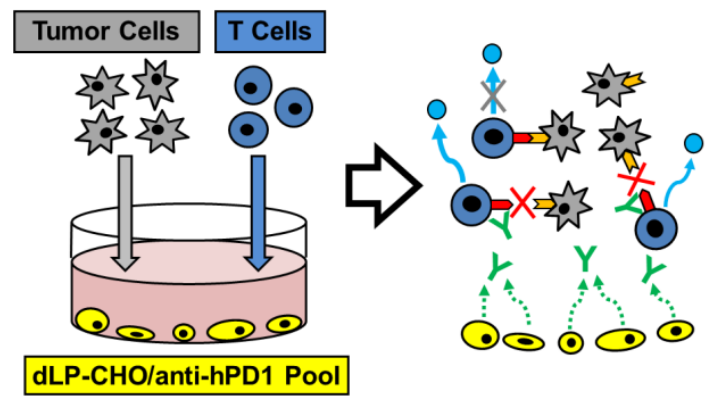

Tumor Cells

T Cells

IFN-Y

$\square$ PD-L1

$\square$ PD-1

\begin{tabular}{|c|}
$\mathbf{A}$ \\
$\begin{array}{c}\text { Anti-hPD1 } \\
\text { mAb }\end{array}$ \\
\hline dLP-CHO Cells \\
\hline
\end{tabular}
C

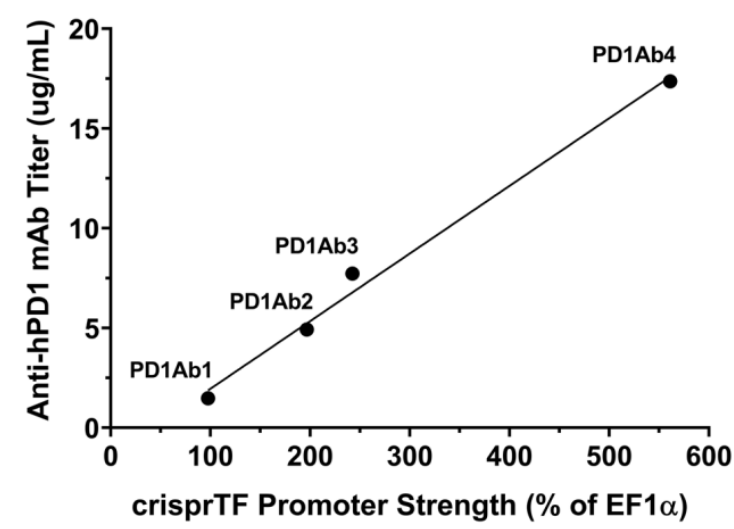

$\mathbf{E}$

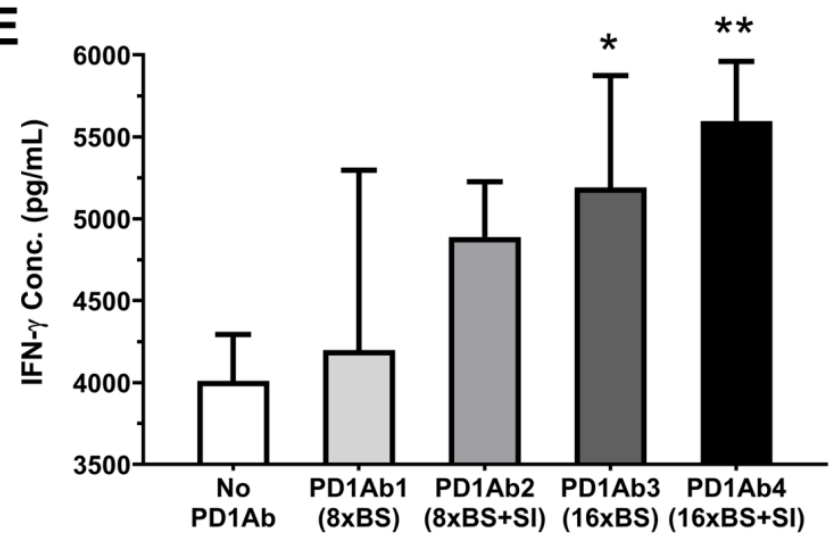

Figure 6. Programmable control of anti-hPD1 secretion and the human T cell anti-tumor response.

(A) Schematic illustration of engineering the anti-hPD1-secreting dLP-CHO cells with sequential and site-specific integration of two payload gene circuits with BxB1 integrase. Clonally sorted EYFP-/EBFP+ dLP-CHO cells with the dLP1-1 site stably integrated with a synthetic gene circuit encoding dCas9-VPR and flanking selection markers were used for the $2^{\text {nd }}$ BxB1-mediated integration. The free dLP1-2 site was integrated with a synthetic gene circuit containing independent TUs that encoded: the 5' flanking puromycin, gRNA10, the light chain and heavy chain of anti-hPD1 driven by the same gRNA10 operators, dCas9-VPR, and the 3' flanking blasticidin linked to dCas9-VPR by a 2A self-cleavage peptide. After selection with four antibiotics for two weeks, dually integrated cells were subjected to pooled cell sorting based on EYFP-/EBFP- signals. Sorted cells were recovered for one passage and then expanded for 1-2 passages under the same selective conditions. (B) Octet mAb titer quantitation showed differential anti-hPD1 secretion 
MIT Confidential

programmed by four distinct configurations: PD1Ab1 (8x BS), PD1Ab2 (8x BS with SI), PD1Ab3 (16x BS), and PD1Ab4 (16x BS with SI). (C) Pearson correlation analysis revealed that anti-hPD1 titers strongly correlated with crisprTF promoter strengths $\left(R^{2}=0.99, p=0.0043\right)$. (D) Schematic diagram of CHO-tumor-T cell co-culture system to evaluate the functionality of anti-hPD1 and to explore the utility of our crisprTF promoter platform for cellular therapy. dLP-CHO cells engineered with one of the above four configurations for anti-hPD1 secretion were first seeded for 48 hours. The control group was seeded with EYFP-/EBFP+ dLP-CHO cells with no integration circuit. Pre-activated human T cells and human ovarian cancer cells (OVCAR8) expressing a surface T-cell engager were subsequently seeded in each well with the attached dLP-CHO cell populations. (E) Quantification of IFN- $\gamma$ concentrations in the media at 24 hrs post-co-culture by ELISA revealed tunable IFN- $\gamma$ production by T cells, with the PD1Ab3 $(p<0.05)$ and PD1Ab4 $(p<0.01)$ groups producing significantly more IFN- $\gamma$ than the control group.

\section{Supplemental Figures and Figure Legends}

\section{Supplemental Figure 1}
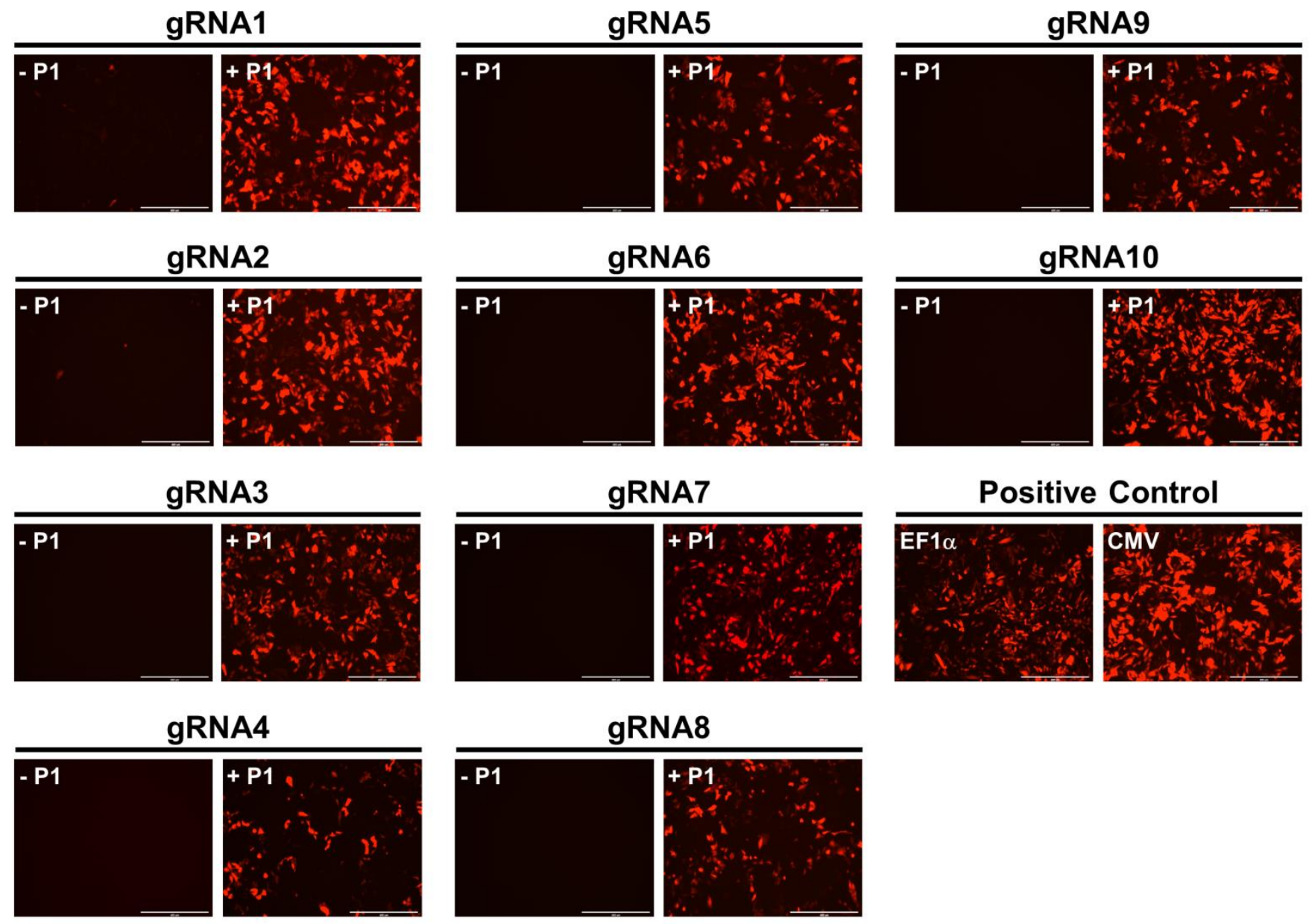

Supplemental Figure 1. Comparison of episomal gene expression levels with 10 distinct gRNA sequences. Each gRNA was paired with a corresponding synthetic operator containing 8x gRNA BS to control mKate transcription. $\mathrm{CHO}-\mathrm{K} 1$ cells were transiently transfected as illustrated in Figure 1C, with gRNA 
MIT Confidential

constitutively expressed by the U6 promoter from plasmid \#1 (P1). Experimental groups were transfected with all 4 plasmids, including P1 (+ P1); negative control groups (no gRNA) were transfected without P1 (P1). Plasmids with mKate expression driven by constitutive promoters (EF1 $\alpha$ or CMV), transfected at the same concentration (250 ng per $1 \times 10^{5}$ cells), served as positive controls. Positive and negative control groups were also supplemented with a dummy plasmid to ensure that all groups had the same total amount of transfected plasmids. Representative fluorescent images revealed a wide range of mKate expression among the 10 different gRNAs at 48 hrs post-transfection. Only gRNA1 and gRNA2 exhibited slight leakage of mKate expression without the presence of $\mathrm{P} 1$.

\section{Supplemental Figure 2}

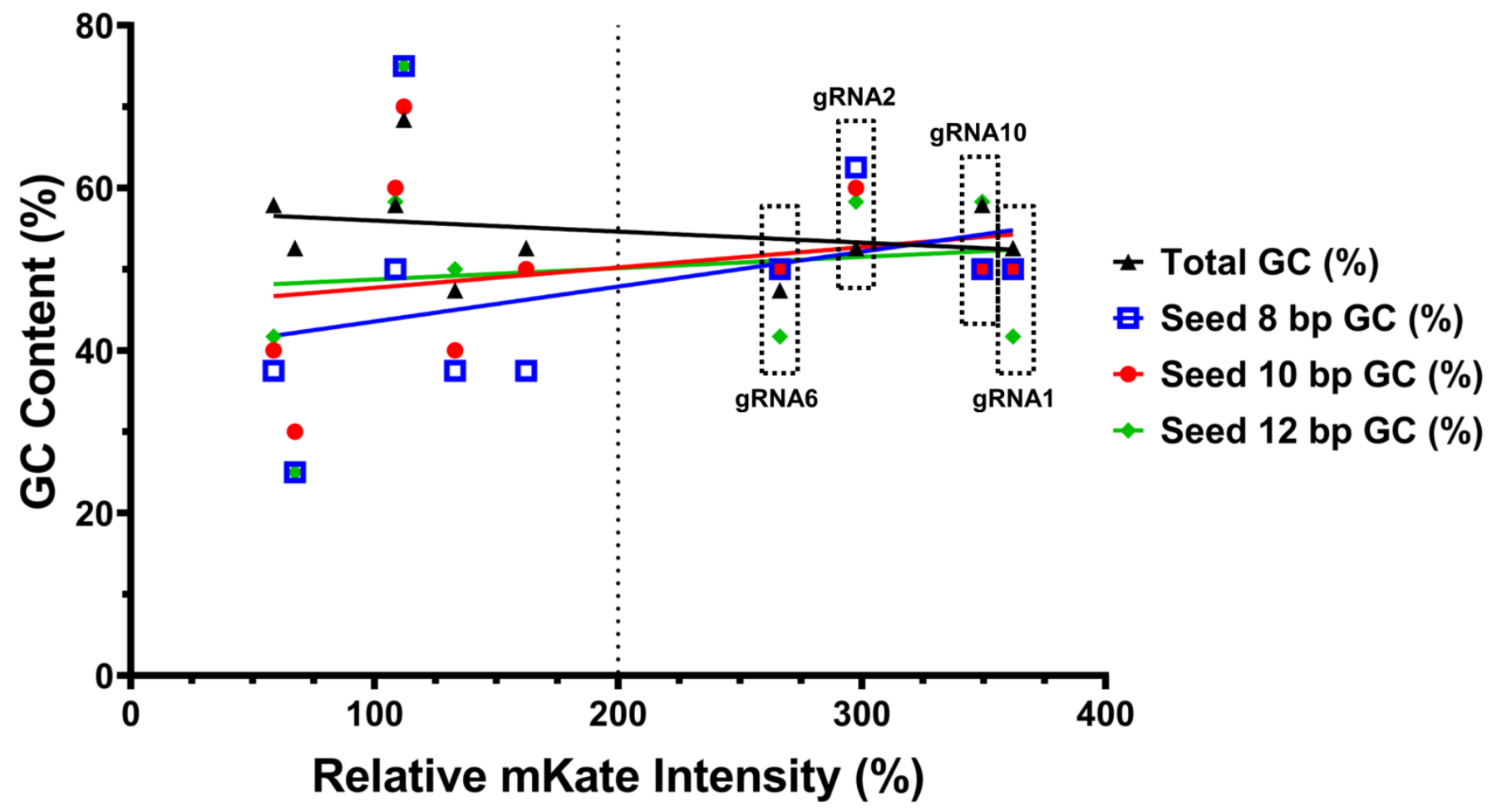

Supplemental Figure 2. Analysis of the correlation between GC content in gRNA seed sequences and gene expression levels. We analyzed the relationship between GC content in gRNA seed 8, 10, and 12 bp sequences and corresponding mKate expression levels among gRNA1-10. Overall, gRNA1, 2, 6, and 10 , which exhibited high mKate expression levels (>200\% of EF1 $\alpha$ promoter), all had $50-60 \%$ GC content in gRNA seed sequences, especially within seed 8-10 bp. 


\section{Supplemental Figure 3}

A
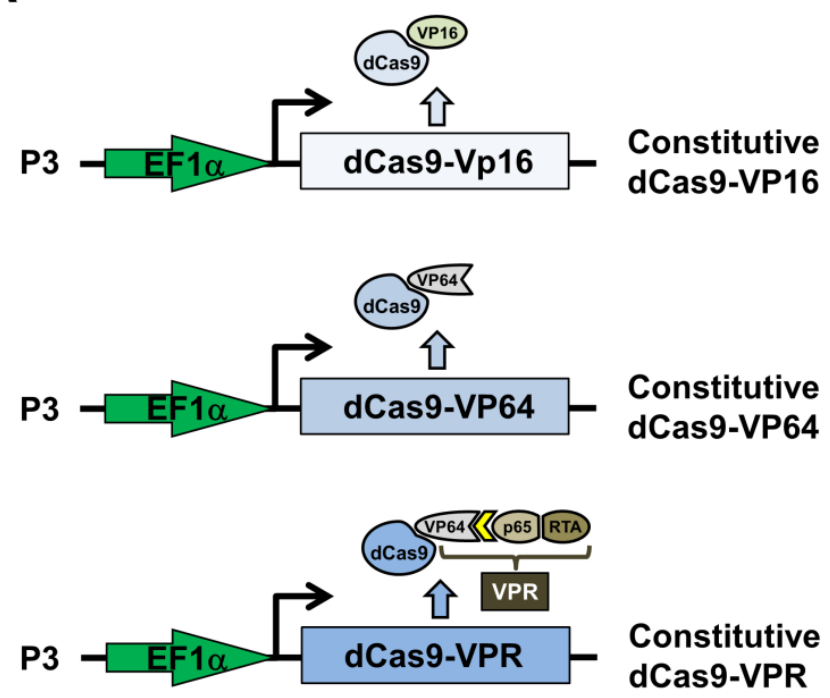

B

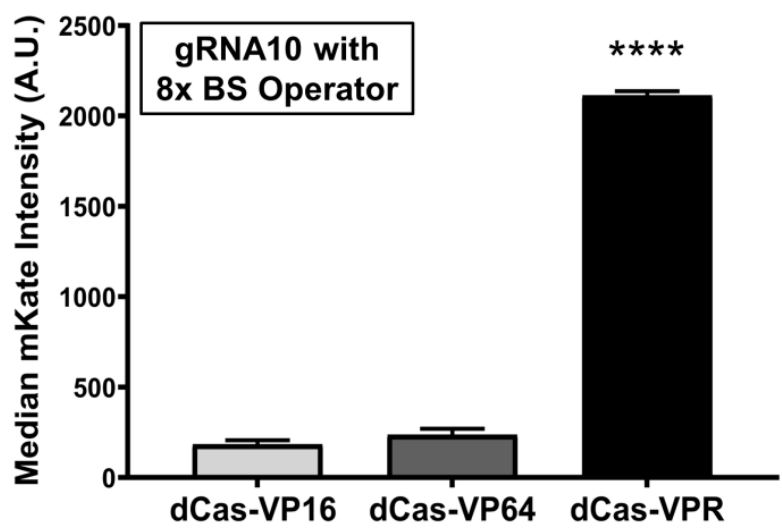

Supplemental Figure 3. Comparison of gene expression levels obtained with 3 crisprTFs. Using gRNA10 (P1) and its 8x BS synthetic operator (P2) as depicted in Figure 1C, we compared mKate expression levels obtained with 3 crisprTFs: dCas-VP16, dCas-VP64, and dCas-VPR. (A) Schematic illustration of Plasmid \#3 (P3) encoding the 3 crisprTFs composed of deactivated SpCas9 (dCas9) with transcriptional activation domains (TADs): dCas-VP16, dCas-VP64, and dCas-VPR. (B) FACS results showed that dCas-VPR had markedly higher median mKate expression than dCas-VP16 (11.5 folds, $p<0.0001)$ and dCas-VP64 (9 folds, $p<0.0001$ ). Data were presented as median mKate intensity of the entire transfected population with artificial units (A.U.). 
MIT Confidential

Supplemental Figure 4
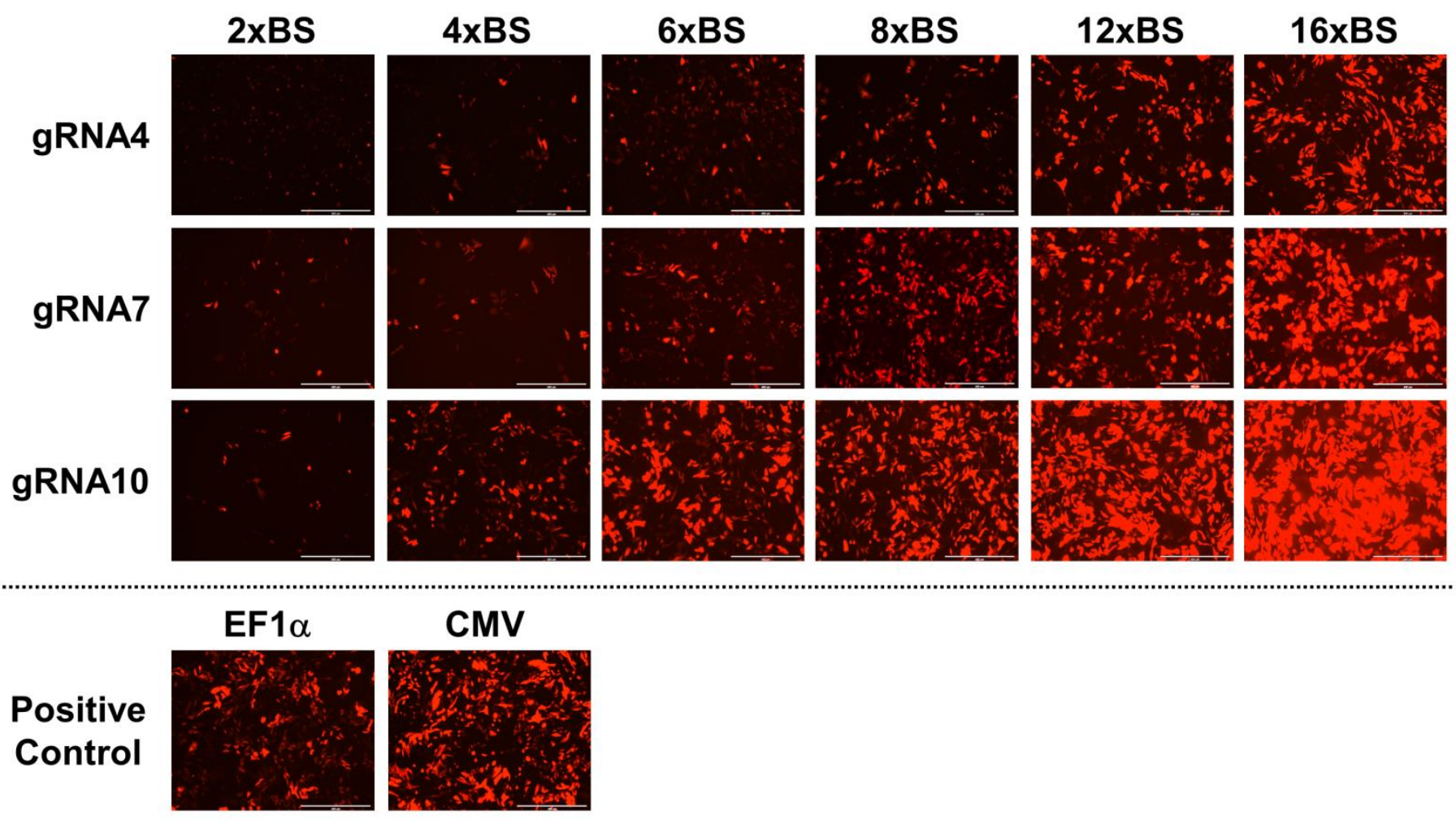

Supplemental Figure 4. Comparison of gene expression levels with 6 distinct synthetic operators containing different numbers of gRNA BS in three gRNA series. $\mathrm{CHO}-\mathrm{K} 1$ cells were transfected as illustrated in Figure 1C, with each gRNA constitutively expressed by the U6 promoter from Plasmid \#1 (P1) and mKate expressed by each synthetic operator from Plasmid \#2 (P2). mKate expression driven by EF1 $\alpha$ and CMV promoters served as positive controls. Representative fluorescent images showed a dramatic range of mKate expression among 6 synthetic operators in all three gRNA series at 48 hrs post-transfection, especially in the gRNA10 series.

Supplemental Figure 5
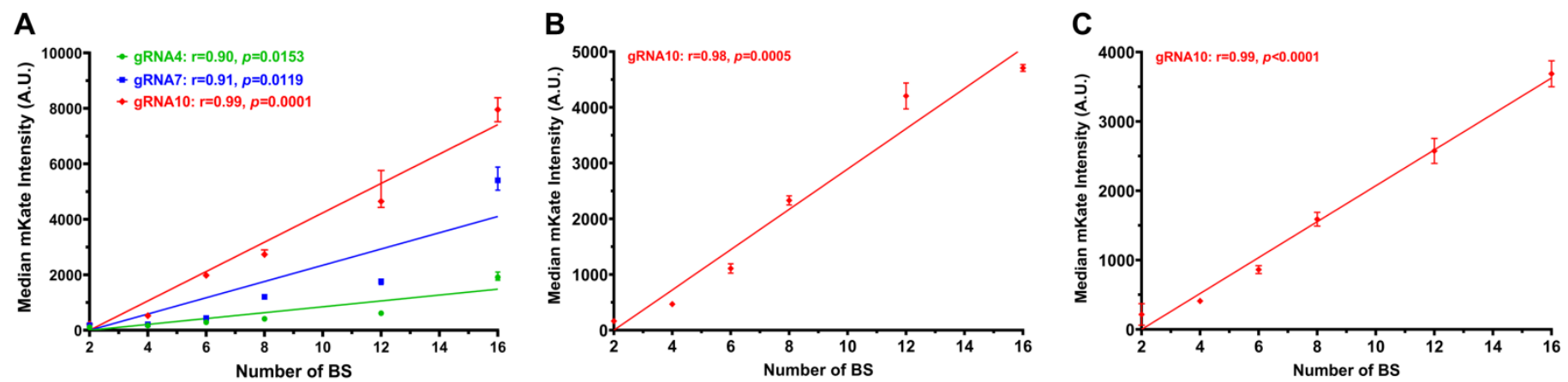

Supplemental Figure 5. Correlation between the number of gRNA BS in the synthetic operator and the gene expression level. Based on the quantitative results from flow cytometry analyses presented in Figure 2, we performed Pearson correlation analysis to reveal the relationship between the number of gRNA BS in each synthetic operator of the three gRNA series and its gene expression level. (A) In CHO-K1 cells, the gRNA4 series had the Pearson correlation coefficient $(r)=0.90\left(R^{2}=0.80, p=0.0153\right)$; the $g R N A 7$ series had $r=0.91\left(R^{2}=0.83, p=0.0119\right)$; and the gRNA10 series had $r=0.99\left(R^{2}=0.98, p=0.0001\right)$. (B) In mouse 
MIT Confidential

C2C12 myoblasts, the gRNA10 series had $r=0.98\left(R^{2}=0.96, p=0.0005\right)$. (C) In human HEK-293T cells, the gRNA10 series had $r=0.99\left(R^{2}=0.99, p<0.0001\right)$. Simple linear regression was performed to plot the graphs.

\section{Supplemental Figure 6}
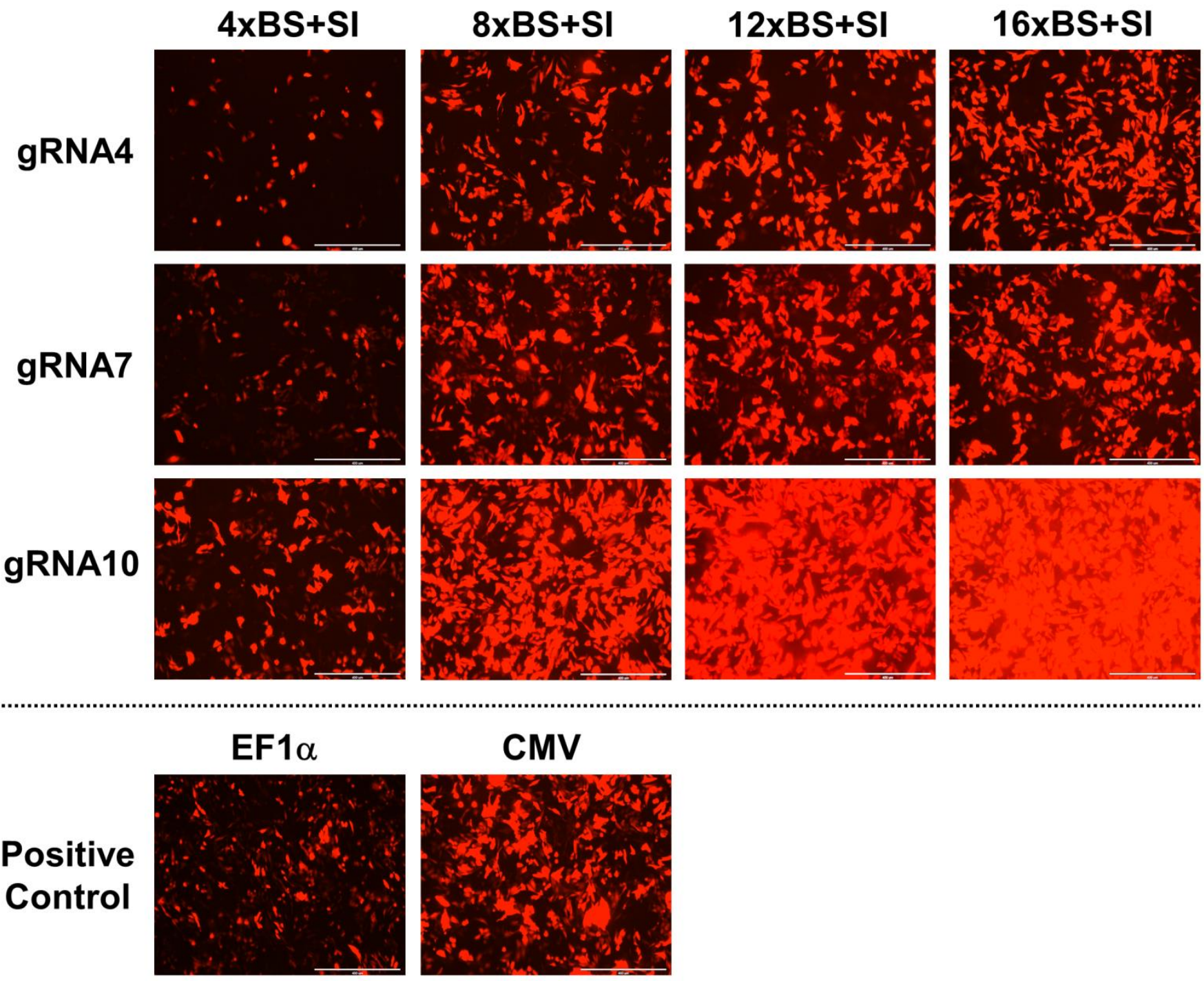

Supplemental Figure 6. Comparison of gene expression levels with the addition of a synthetic intron (SI) in 4 distinct synthetic operators in three gRNA series. $\mathrm{CHO}-\mathrm{K} 1$ cells were transfected as illustrated in Figures 1C and 3D, with each gRNA expressed constitutively from P1. mKate was expressed by each synthetic operator (P2) with the presence of an SI at the 5' UTR of the mKate gene. mKate expression driven by EF1 $\alpha$ or CMV promoters served as positive controls. Representative fluorescent images revealed marked increases in mKate expression with the addition of the SI in 4 different synthetic operators of each gRNA series at $48 \mathrm{hrs}$ post-transfection. The increment was particularly noteworthy in the gRNA10 series when compared with mKate signals expressed by the same synthetic operators without the SI (data shown in Supplemental Figure 4). 
MIT Confidential

\section{Supplemental Figure 7}

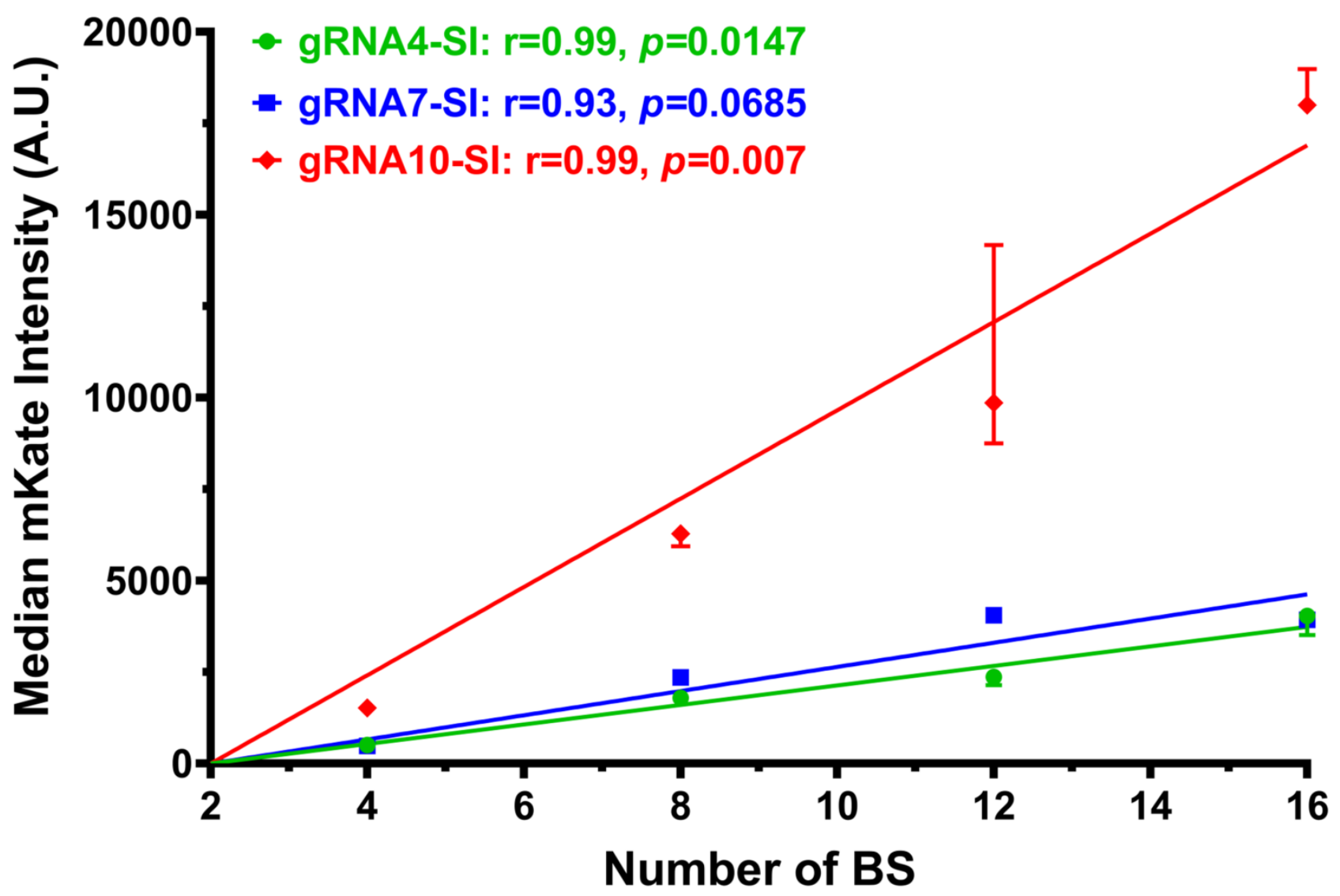

Supplemental Figure 7. Correlation between the number of gRNA BS and the gene expression level with the addition of a synthetic intron (SI). Pearson correlation analysis uncovered the relationship between the number of $g R N A B S$ in the synthetic operator and the associated gene expression level with the presence of an $\mathrm{SI}$ at the 5' UTR of the target gene. The gRNA4-SI series had the Pearson correlation coefficient $(r)=0.99\left(R^{2}=0.97, p=0.0147\right)$; the gRNA7-SI series had $r=0.93\left(R^{2}=0.87, p=0.0685\right)$; and the gRNA10-SI series had $r=0.99\left(R^{2}=0.99, p=0.007\right)$. Simple linear regression was performed to plot the graph. 


\section{Supplemental Figure 8}

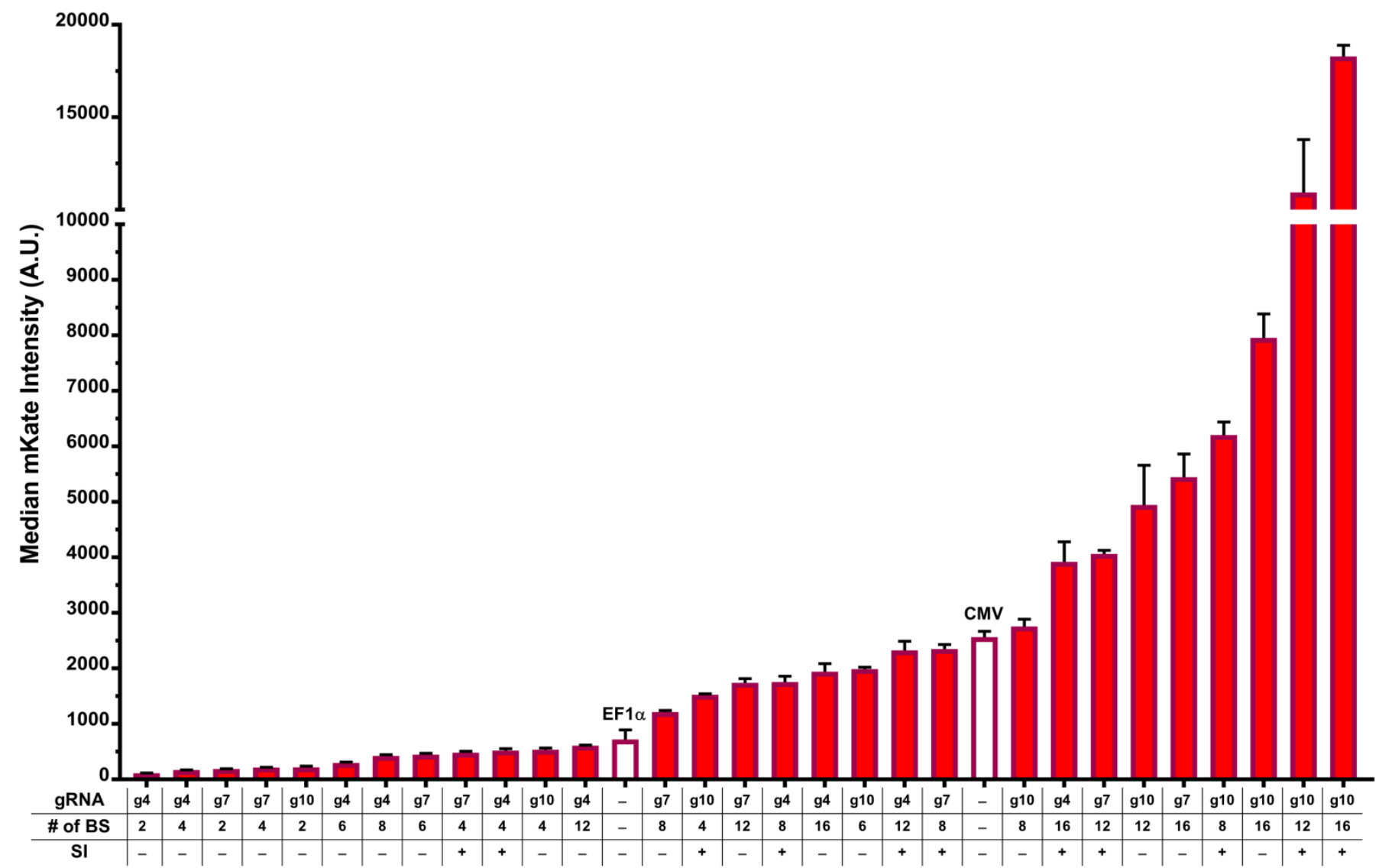

Supplemental Figure 8. Summary of construct compositions and corresponding gene expression levels. Compositions and gene expression levels of constructs from gRNA4 (g4), gRNA7 (g7), and gRNA10 (g10) series that were tested episomally, with or without the synthetic intron (SI). EF1 $\alpha$ and CMV promoter controls are represented by empty bars.

Supplemental Figure 9

A

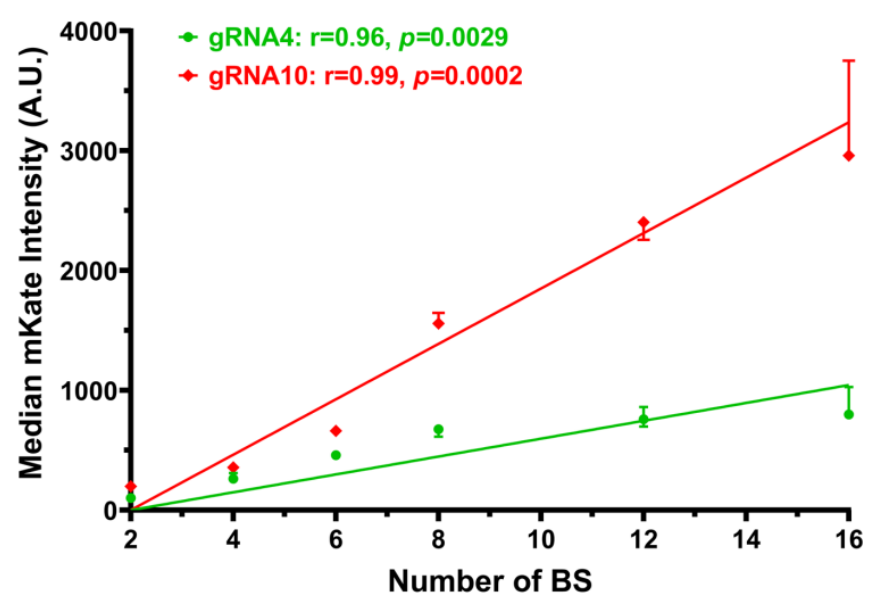

B

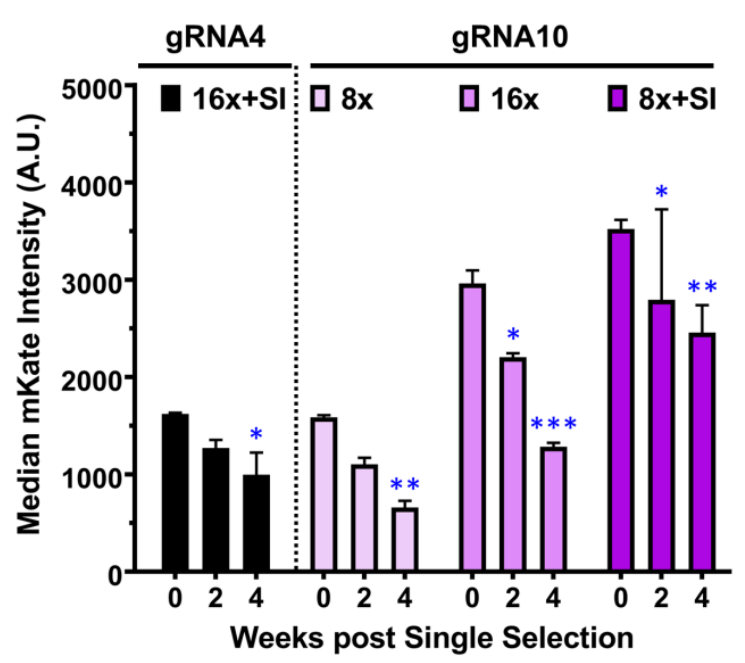

Supplemental Figure 9. Analyses of chromosomally integrated crisprTF circuits. (A) Pearson correlation analysis showed the relationship between the number of gRNA BS in the synthetic operator and 
MIT Confidential

the associated gene expression level when gene circuits were chromosomally integrated. The gRNA4 series had the Pearson correlation coefficient $(r)=0.96\left(R^{2}=0.91, p=0.0029\right)$, and the gRNA10 series had $r=0.99$ $\left(R^{2}=0.98, p=0.0002\right)$. Simple linear regression was performed to plot the graph. (B) After puromycin selection, representative circuit integrants from both gRNA4 (16x BS with SI) and gRNA10 (8x and16x BS without $\mathrm{SI}$, and $8 \mathrm{x}$ BS with $\mathrm{SI}$ ) series all exhibited notable, progressive decreases in mKate expression levels during the additional 4 weeks of culturing $\left({ }^{*} p \leq 0.05 ;{ }^{* *} p \leq 0.01 ;{ }^{* * *} p \leq 0.001\right)$.

\section{Supplemental Figure 10.}

A

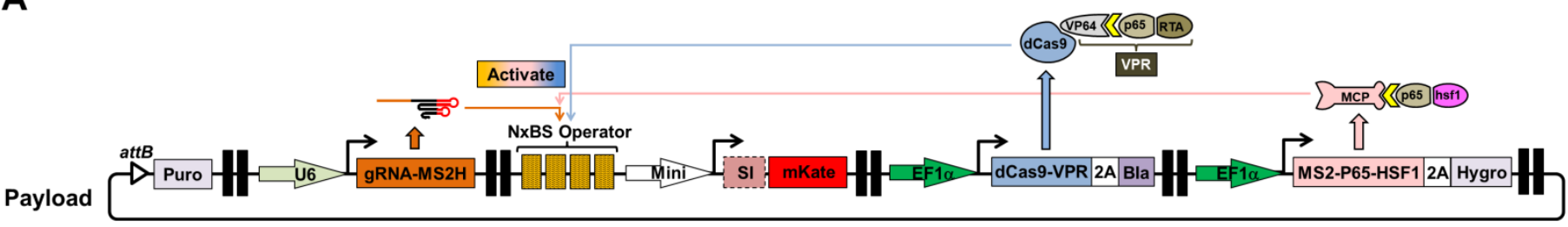

B

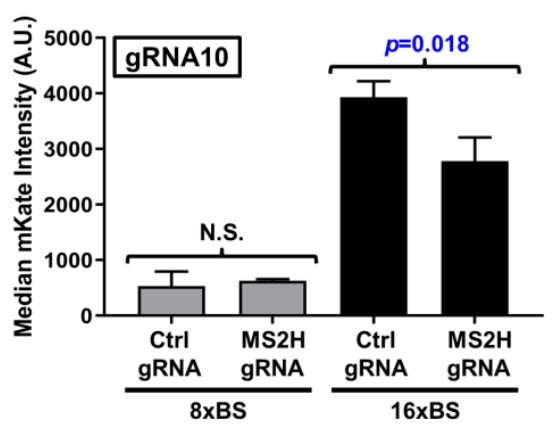

Supplemental Figure 10. Investigation of the synergistic effect between SAM and dCas-VPR at the chromosomal level. (A) A schematic illustration of the gene circuit for chromosomal integration in sLP-CHO cells. The circuit constitutively co-expressed SAM, including gRNA with MS2 hairpins and MCP-p65-hsf1, and dCas9-VPR as well as 3 selection marker genes, including the 5' flanking puromycin and the 3' flanking blasticidin (associated with dCas9-VPR gene using a self-cleaving P2A peptide) and hygromycin (associated with MCP-p65-hsf1 gene using P2A). (B) sLP-CHO cells were transfected with the gene circuit and a plasmid expressing BxB1 integrase. Following recovery in complete culture medium without any antibiotic for 3 days, cells were then selected with puromycin, blasticidin, and hygromycin for 10 days. After this triple selection, flow cytometry was performed to evaluate mKate expression levels. The results showed no synergistic effect between SAM and dCas-VPR with the chromosomally integrated gRNA10 8xBS operator $(p>0.05)$. mKate signals significantly decreased when SAM and dCas-VPR were acting together with the gRNA10 16xBS operator $(p=0.018)$. 
MIT Confidential

\section{Supplemental Figure 11}

A

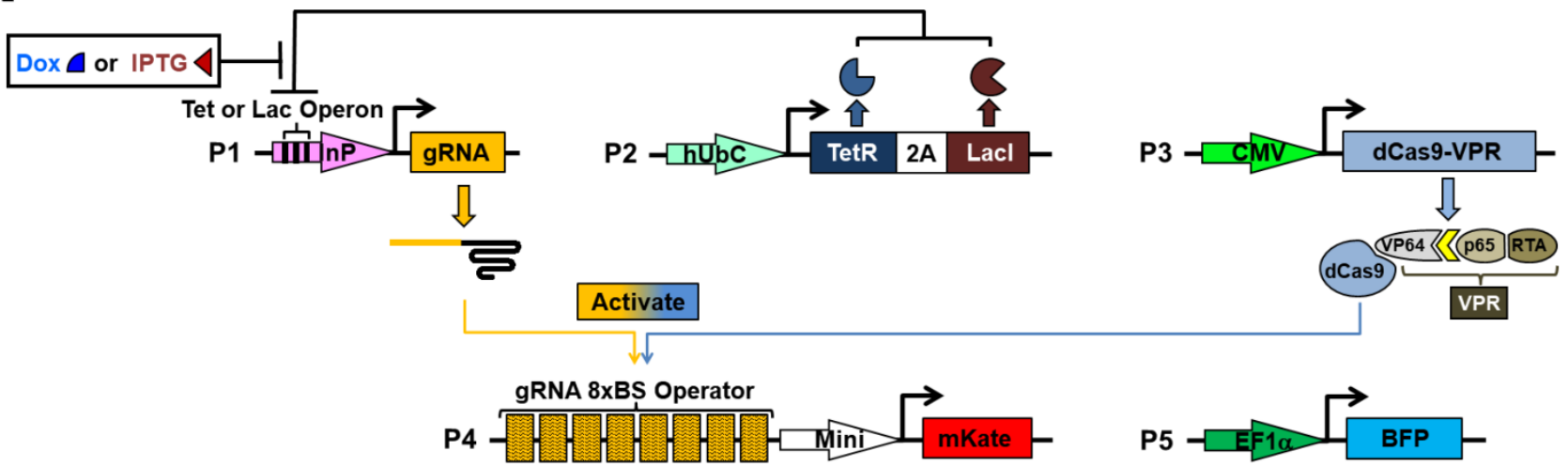

B
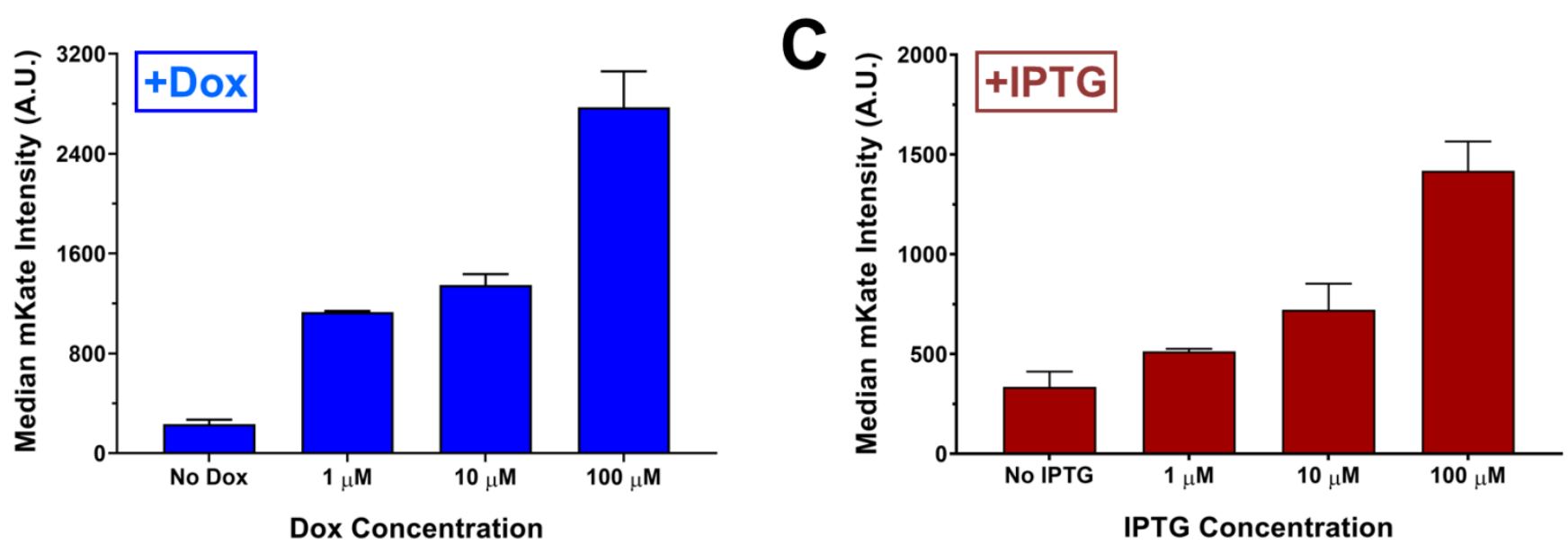

Supplemental Figure 11. crisprTF promoter with small molecule-inducible gRNA expression. (A) To equip crisprTF promoters with an added tier of controllability, we developed inducible switches with

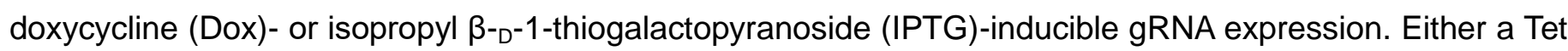
or a Lac operon was imbedded into a DNA polymerase III (pol III) promoter driving gRNA expression to render inducibility. Without an appropriate small molecule inducer (Dox or IPTG), the Tet repressor or Lac inhibitor (both constitutively expressed from a single plasmid using a self-cleaving P2A peptide) bound to the Tet or Lac operon, respectively, and repressed gRNA expression. In the presence of Dox or IPTG, the Tet repressor or Lac inhibitor did not bind to the respective operon, permitting transcription. (B) Titration analysis of the Dox-inducible crisprTF promoter unveiled incrementally increased mKate expression with increased Dox concentration; the highest expression level was seen with $100 \mu \mathrm{M}$ Dox. (C) Titration analysis of the IPTG-inducible crisprTF promoter revealed incrementally increased mKate expression with increased IPTG concentration; the highest expression level was seen with $100 \mu \mathrm{M}$ IPTG. 